\title{
L'octogone des événements émotionnels : un modèle pour comparer la gestion des émotions à un âge précoce
}

\author{
Éva Feig',* \\ EA 1339 LiLPa, Université de Strasbourg, 67000 Strasbourg, France
}

\begin{abstract}
Résumé. Pour que les adultes travaillant dans des crèches ou écoles maternelles bi-plurilingues puissent comprendre le comportement corégulatif de leurs collègues, issus d'une autre langue-culture, nous développons un nouveau modèle sous forme d'un diagramme Kiviat qui se fonde sur ce qu'on peut observer comme adulte au niveau intra-individuel pendant une situation de charge émotionnelle vécue par l'enfant (ici nommé événement émotionnel). Le modèle prend en considération les différentes dimensions qui caractérisent l'événement émotionnel vécu par l'enfant de même que sa gestion par l'adulte. Il visualise l'instant observé selon huit axes, à savoir : la valence émotionnelle, l'intensité du ressenti enfantin, la modalité du traitement offert par l'adulte, le degré de verbalisation du ressenti offert par l'adulte, la familiarité de l'enfant avec les mots utilisés par l'adulte, la sémiotisation des émotions du côté adulte, la focalisation plus ou moins centrée sur les émotions de l'enfant et la situationalité ou le degré d'induction situationnelle du vécu. Sur la base des observations faites dans quatre crèches franco-allemandes de l'espace du Rhin supérieur en 2018, il est possible de montrer l'applicabilité pratique du modèle pour des études interculturelles. De plus, l'abstraction des figures qui reflètent la stratégie de co-régulation des émotions mise en place par chaque pédagogue permet d'identifier des congruences comportementales transculturelles entre les professionnels francophones et germanophones qui ont réussi à établir une culture pédagogique partagée, voire une approche « interdidactique » selon Puren [7].
\end{abstract}

\begin{abstract}
For day-care centre and preschool teachers in bi-plurilingual settings to understand their colleagues' co-regulative behaviour, we develop a new model in the form of a Kiviat diagram that accounts for the adults' emotion management and intraindividual perception of the child's emotional load during what we call an emotional event. The eight parameters taken into consideration are as follows: arousal, emotional value and intensity of the child's emotion, the modality of the adult's interactions, its degree of verbalisation of the emotion, the child's familiarity with the words used by the adult, the adult's semiotisation of the emotion, his or her childcentredness and the context-drivenness of the co-regulated event. By means of the observations made in 2018 in four French-German day-care centres
\end{abstract}

\footnotetext{
*evafeig@gmail.com
} 
situated in the region of the Upper Rhine it is possible to demonstrate the model's practical applicability for intercultural studies. The silhouettes reflecting the co-regulation strategy of each pedagogue allows us to identify transcultural congruencies in the behaviour of French- and Germanspeaking teachers who have managed to implement a shared pedagogical culture, i.e. an "interdidactic" approach according to Puren [7].

\section{Introduction : Pourquoi encore un modèle ?}

Si l'on travaille dans le champ de la petite enfance et, plus concrètement, avec des structures d'accueil à vocation bi-plurilingue où l'on vise expressément une inculturation double du jeune enfant, les émotions sont partout et au cœur de tout ce qui est fait. Néanmoins, et bien qu'elles paraissent très banales, les émotions sont un champ très vaste, très difficiles à saisir et profondément enracinées dans une culture concrète [3, p.11]. Ainsi, et bien que certaines réactions physiologiques se trouvent hors de l'influence de la culture, c'est elle qui détermine comment les émotions sont vécues et communiquées, respectivement, comment on est censé les percevoir et exprimer [4, p. 106] [cf. 5, 6]. Pour aider les professionnels à accompagner les processus de socialisation affective avec la sensibilité nécessaire à l'égard des habitudes de l'autre et pour les orienter dans l'établissement d'une culture pédagogique partagée - ce que nous nommons " interdidacticité » selon Puren [7, p. 491] -, il est nécessaire de se distancer des résultats obtenus en laboratoire $[8,9,10,11,12,13,14]$, en recherche développementale monolingue $[15,16,17,18,19,20]$ ou dans des études lexicographiques $[21,22,23]$ ou lexico-centrées $[24,25,26,27,28]$ pour procéder à la découverte des patrons interactionnels du terrain. Ce changement de paradigme s'avère d'autant plus nécessaire qu'il faut rappeler avec Clore et Ortony [29, p. 368] que beaucoup d'études qui analysent le vocabulaire affectif partent d'un corpus de mots dits émotionnels sans avoir défini préalablement les critères exacts qui justifient l'exclusion ou inclusion des mots. Pour combler cette lacune, nous suivrons des chercheurs interactionnistes comme Quignard et al., Plantin, Plantin et al., Polo et al. et Cosnier [30, 31, 32, 33, 34] et nous nous concentrerons sur les préférences et habitudes qu'on peut observer in situ. Les nouvelles connaissances qui découlent d'une telle approche du terrain permettront de comparer comment les acteurs professionnels de l'accueil de la petite enfance co-régulent les émotions du jeune enfant pendant ce que nous appelons un " évènement émotionnel » et ceci dans un environnement bi- ou plurilingue. De plus, cette recherche nous fournira un premier inventaire des termes qu'ils leur apprennent pour nommer ce qu'ils ressentent. Ce but s'avère d'autant plus important en vue de l'affluence des recherches hautement scientifiques sur les émotions des nourrissons qui ne peuvent pas être transférées du setting expérimental au terrain, mais aussi compte-tenu des publications de vulgarisation - comme celles de Filliozat -, qui offrent aux intéressés des leçons et des petits exercices pratico-pratiques qui simplifient exagérément et généralisent trop pour servir de base pour une formation continue des professionnels déjà bien formés dans le champ de la psychologie du petit enfant et du travail de l'attachement.

Avant de pouvoir générer un tel modèle qui se prête à une analyse multifactorielle et comparative, il faudra quand même déterminer les défis théoriques et ainsi répondre aux questions suivantes :

1. Que définit-on comme « émotion »?

2. Comment peut-on repérer et décrire un évènement émotionnel et ce, plus particulièrement, chez le jeune enfant?

3. Comment classifier les événements d'implication émotionnelle dans un contexte biplurilingue sans se laisser obnubiler par le réseau lexical de l'une des langues impliquées ?

S'il y a des réponses pertinentes, il sera possible de comparer 
a) la manière dont les éducatrices/éducateurs nomment, c'est-à-dire interprètent et catégorisent des événements comparables (comment les mettent-ils/elles en mots dans une langue et dans l'autre ?) et

b) la manière dont les éducatrices/éducateurs gèrent des situations analogues concrètement (p.ex., pour calmer le chagrin d'un enfant pendant l'accueil du matin, est-ce qu'ils/elles verbalisent tout ou se contentent-ils/elles de stratégies non-verbales ?).

Le modèle qui en résultera permettra de mener des études qui pourront contribuer à une harmonisation des premières expériences émotionnelles faites dans les deux langues offertes dans les structures d'accueil de la petite enfance. Ainsi, on pourra prévenir une mémorisation à long terme des déclencheurs fondamentaux ou traumatiques qui éliciteront dès lors des croyances stéréotypiques qui seront inaccessibles aux processus cognitifs appris ultérieurement [35, p. 34-35]. Cet objectif s'avère d'autant plus important qu'il faut rappeler avec [36, p. 55] qu'au début de la vie, nous partageons tous les mêmes patrons psychophysiologiques concernant le traitement des émotions (pour le concept d'une multicompétence émotionnelle unique des individus bi-plurilingues cf. [5, p. 2] et [37]). C'est seulement après que commence l'effet modélisateur de la culture, donc la socialisation du nourrisson, dont $[\mathrm{u}] \mathrm{n}$ des objectifs [...] est d'apprendre à l'enfant comment utiliser la langue pour produire des énoncés qui aient un sens, de façon à la fois efficace et acceptable pour la communauté » [38, p. 17] [cf. 39, p. 182]. Si ce processus débute, néanmoins, « dès la naissance puisque la manière dont on s'adresse ou pas à l'enfant est régie et organisée par la culture dans laquelle on s'insère », il serait souhaitable que les professionnels de l'accueil du jeune enfant soient sensibilisés à cet enjeu pour mieux accompagner l'entrée du jeune enfant - même de celui qui est monolingue de naissance - dans le monde bilingue-biculturel.

\section{Ancrage théorique et cadre définitoire}

Pour rester ouverte à la diversité des savoir-dire, savoir-faire et savoir -être de chaque langue-culture, cette étude choisit une perspective onomasiologique en partant du phénomène observable pour en inventorier les différentes stratégies de dire et faire. Ce faisant, notre questionnement s'ancre, d'une part, dans le champ de la psychologie du jeune enfant, notamment dans l'éducation et, d'autre part, dans le champ de l'ethnolinguistique. De cette façon, il peut profiter des connaissances et modèles de différentes disciplines pour en emprunter les composants et méthodes qui se prêtent à une application in vivo pour comprendre et classifier la réalité des interactions humaines.

Au vu de l'âge précoce des enfants impliqués (de 10 semaines à 3 ans), il va de soi que toute approche partant d'une théorie de l'évaluation cognitive (cognitive appraisal) comme base définitoire exclusive des émotions sera difficile à mettre en place ici, car l'accès à la classification mentale du ressenti à travers le langage des émotions est pratiquement bloqué. Ce défi est particulièrement aigu parce qu'il faut admettre avec Ortony, Clore et Collins [40, p. 8] que bien que les émotions ne soient pas des entités linguistiques, on y accède primordialement à travers le langage. Au-delà de ce défi particulier de la tranche d'âge ciblée, nous devons faire face à la problématique générale que les émotions se présentent sous forme de black box [41, p. 244, p. 246 Figure 11.1] [cf. 42, p. 5], dont le contenu doit être déduit des indices comportementaux, verbaux et physiologiques [43, p. 174, 176] [44, p. 127] [40, p. 8-12]. De plus, et lié de nouveau au monde de la petite enfance, il faut être conscient qu'au début de la vie, le fait d'expérimenter un événement émotionnel $†$ et de réagir à sa cause sont

$\dagger$ Cf. la théorie des événements affectifs (Affective Events Theory) de Weiss et Cropanzano [1, p. 11] qui place le focus également sur les événements singuliers et concrets comme déclencheurs ou causes d'une réaction affective. L'argumentation de Scherer (2005: 700) va dans le même sens quand il écrit : 
deux circuits neuronaux inséparables. Ainsi, dans l'ontogenèse de l'être humain, on peut supposer une chronologie relative qui commence par des processus bottom-up [45, p. 88], caractérisés par les réflexes et les anciens programmes d'auto-préservation du cerveau archaïque [46, p. 163), et qui continue à s'enrichir par des processus top-down [45, p. 89-90) qui permettent un contrôle de plus en plus raffiné sur ces réactions innées et, donc, la gestion des émotions à l'aide de l'évaluation cognitive et du langage. La langue et donc, le contrôle sur les émotions, ne s'entremêlent que peu à peu [41, p. 243]. Comme cette « cognitivisation » du vécu et le réseau lexical et métaphorique représentant les émotions se développent à l'aide de ${ }^{\ddagger}$ et en interaction multimodale avec [39] [36, p. 55] d'autres acteurs de l'environnement du jeune enfant $[47,48][39, \text { p. 165-166 }]^{\S}$ - ici des éducateurs parlant deux langues différentes - et comme nous souhaitons étudier justement les concepts verbaux et interactionnels qu'ils apprennent aux enfants, l'identification d'un événement émotionnel devra se fonder primordialement sur les autres canaux ou output systems [41, p. 244), comme le sont le comportement et les réactions physiologiques [46, p. 163) qui sont intrinsèquement liées [49, p.185]. La présente étude part, par conséquent, d'une définition de l'émotion qui est empruntée à des études psychologiques physio-comportementales de l'émotion comme celle de Lajante et Droulers $\left[43\right.$, p. 174, 176] ${ }^{* *}$, qui suivent Grandjean et al. [50, p. 485] en déterminant l'émotion (primaire) comme un

\begin{abstract}
épisode dynamique qui implique un processus de changement continu dans tous les sous-systèmes (cognition, motivation, réactions physiologiques, expressions motrices) pour s'adapter avec flexibilité aux évènements pertinents et aux conséquences potentiellement importantes pour un individu » [50, p. 485, traduit et cité par 43, p. 173].††
\end{abstract}

Pour plus de précision, on devrait y ajouter également la dimension vocale $\$$ qui est d'importance fondamentale dans les relations interculturelles [19] et qui a été étudiée en détail, entre autres, par Moulin [51, p. 156], Devillers et al. [52, p. 129], Abitbol [53] et Prat [54, p. 29 s.]. Le terme émotions est alors l'hypéronyme de toute une série de phénomènes visibles, audibles, palpables et qu'on peut parfois même sentir si l'on est assez proche de la personne qui expérimente l'émotion, selon ce que nous apprend Hall [55, p. 70s.). Néanmoins, et au contraire de ce que l'on peut faire dans les études menées en laboratoire ou dans le contexte des recherches néonatales en clinique, les indices corporels comme la fréquence cardiaque $\left[43\right.$, p. 174-175) ou la réaction électrodermale (RED) ${ }^{\S \S}[56$, p. 150) ne peuvent pas être utilisés comme marqueurs objectifs de l'arousal afin d'identifier des épisodes émotionnels chez le jeune enfant passant la journée dans une crèche bilingue. Le même constat vaut pour la méthode de l'autoévaluation (self-report) [40, p. 9] qui est

\footnotetext{
« emotions are generally elicited by stimulus events. By this term I mean that something happens to the organism that stimulates or triggers a response after having been evaluated for its significance».

$¥$ Cf. le concept vygotskien de la zona blizhaichego razvitia ou zone du développement le plus proche $[57$, p. 60] qui décrit le «parcours développemental qu'il [l'enfant] est sur le point d'accomplir » [58, p. 136].

$\S$ Ainsi Meltzoff et Moore ont pu montrer que déjà 42 minutes après sa naissance, un bébé est capable et essaiera d'imiter les mouvements d'un autre humain adulte [59, p. 779].

${ }^{* *}$ Cf. Ortony et Clore [44, p. 127], Damaisio et Larsonneur [60], mais aussi des définitions proposées par des praticiens comme, par exemple, Gervais [61, p. 7].

$¥$ I† Concernant l'acceptation générale d'une définition multicomponentielle entre les chercheurs, voir aussi, par exemple, García-Prieto et al. [62, p. 197], Gross et Thompson [42, p. 4-5], Frijda [63, p. 264] ou Celeghin et al. [64, p. 2].

$\$$ Cf. aussi les six composants de l'émotion décrits par Fontaine et al. [65, p. 1051-1054].

$\$$ Lajante et Droulers [43, p. 175] parlent d'activité électrodermale (AED).
} 
l'instrument le plus utilisé quand on travaille avec des adolescents ou des adultes. Dans le contexte de la petite enfance, cet instrument est simplement inutile [66, p. 252].

La perspective intra-individuelle (within-individual level) [49, p. 177] étant non viable, il faudra alors favoriser une approche inter-individuelle (between-individual level) [49, $\mathrm{p}$. $177]^{* * *}$ comme celle que propose l'ethnographie et, plus précisément, l'observation participante. Celle-ci vise à documenter tous les symptômes et facteurs contextuels qui peuvent éclairer une situation concrète, se prêtant ainsi à l'analyse multifactorielle que nous envisageons de faire. Ainsi, l'identification d'un événement émotionnel vécu par l'enfant prendra en considération, premièrement, les indices visuels, auditifs et tactiles, par exemple quand l'enfant se raidit, quand il a des mains froides ou commence à transpirer fortement, etc. Cette méthode peut se justifier, de plus, par la similarité croisée (cross-modal similarity) entre les résultats des différents types de test documentés par Nummenmaa et Saarimäki [67, p. 5, Fig. 1]. Deuxièmement, il tiendra compte de toute sorte d'interaction avec l'environnement ${ }^{\dagger \dagger \dagger}$ - dont s'aperçoivent tantôt la chercheure, tantôt les acteurs professionnels. En ce qui concerne la définition d'interaction dans ce contexte nous suivons Kerbrat-Orecchioni [68, p. 216] en postulant :

Pour qu'on ait affaire à une seule et même interaction, il faut et il suffit que l'on ait un groupe de participants modifiable mais sans rupture, qui dans un cadre spatiotemporel modifiable mais sans rupture, parlent d'un objet modifiable et sans rupture.

L'interaction est donc une «entrée en contact intentionnelle avec un sujet ou objet coprésent dans l'épisode émotionnel vécu afin d'y exercer une influence » [69, p. 55]. Néanmoins, cette deuxième idée de vouloir « changer en échangeant » [69, p. 55] focalisée sur les échanges et négociations verbales devra être étendu à d'autres types d'échanges non verbaux pour être en phase avec notre recherche. Par conséquent, nous définirons l'émotion comme perception d'une déviation d'apparence et de comportement relativement à ce qu'on perçoit ou définit comme " ligne de base » dans un micro-contexte donné (concept de " figure-fond »). Ces déviations se peuvent présenter sous forme d'une différence "par présence », c'est-à-dire sous forme d'un comportement qui diffère activement de ce que l'on peut attendre (p.ex. l'enfant hurle, tape, mord, se jette sur le sol, devient tout rouge, pâlit, pleure à chaudes larmes, glousse de plaisir, etc.), ou bien elles peuvent se présenter sous forme d'une différence "par absence», quand l'enfant ne montre pas le comportement prévisible (p.ex. quand il reste sans bouger, sans parler, sans entrer en interaction pendant un certain temps et que cela interpelle son environnement).

\section{Vers un nouveau modèle interculturel : les paramètres (ou axes) à prendre en considération}

Afin de rendre compte de tous les facteurs qui déterminent la perception d'un événement émotionnel chez le jeune enfant ainsi que sa gestion par l'adulte et ceci sans recourir aux stéréotypes de l'une des cultures étudiées $\$+$, nous devons trouver une représentation qui prenne en considération la complexité du phénomène et de l'interaction à décrire et qui soit, dans l'idéal, abstraite, claire et auto-explicative. Nous partons alors avec Fontaine et al. [65, p. 1050] de la constatation que le monde des émotions n'est pas bidimensionnel et, à la

\footnotetext{
*** Pour le problème de l'observabilité des émotions dans le discours cf. aussi Micheli, Hekmat et Rabatel [75].

I†† Pour une analyse plus ample des « émotions dans les interactions » cf., par exemple, Plantin, Doury et Traverso [32].

林 Pour l'ancrage profondément culturel des expressions des émotions cf. aussi, par exemple, KwapiszOsadnik [76] et Pavlenko [77].
} 
recherche des idées utiles, nous tournons d'abord vers les modèles dimensionnels comme celui de Berthelon [70, p. 27 s.], ou des modèles componentiels comme celui de l'hexagone des émotions élaboré par Sprengelmeyer et al. [71, p. 1653]. Cette représentation hexagonale de la confusabilité des émotions fondamentales [14, p. 37, figure 2.1] se base sur les photos tirées du pool des expressions faciales d'Ekman et Friesen [72] qui ont été consultés dans sa visualisation chez Daudelin-Peltier [73, p. 74, Figure 15] et elle a été dessinée, surtout, pour permettre une classification des expressions du visage qui soit plus nuancée (p.ex. joiesurprise) et qui prenne en considération la possibilité d'un état émotionnel plus complexe que celui des émotions « pures » définies par Ekman/Friesen (cf. dans ce contexte « The Emotion Hexagon Test » développé et utilisé dans le manuel de Sprengelmeyer et al. [14]). Elle nous servira de source d'idée d'une représentation n-gonale, mais pendant que le modèle de Sprengelmeyer et al. a été dessiné expressément pour montrer la proximité, donc " confusabilité » de certains expressions faciales émotionnelles (hexagonal representation of confusabilities of basic émotions en anglais), nous cherchons une visualisation qui soit composée d'axes indépendantes. Une deuxième source d'inspiration pour un tel modèle sont les axes du modèle circomplexe de l'affect de Russel (Russell 1980) ou les composants de l'émotion déterminés par Scherer [74, p. 698, Tableau 1), mais nous devrons compléter ces modèles par les différents facteurs qui déterminent l'interaction co-régulative entre pédagogue et jeune enfant afin d'adapter la visualisation à notre terrain. Dans ce but, nous proposons de prendre en considération huit dimensions qui caractérisent ces événements émotionnels :

\section{AXE 1: La valence émotionnelle (positive - neutre - négative) :}

Elle décrit comment le vécu, notamment l'excitation du système nerveux, devrait être évalué par le jeune enfant et elle s'organise entre les deux pôles [ÉMOTION POSITIVE] et [ÉMOTION NÉGATIVE] [78, p. 174]. La dimension neutre ou ambivalente (surprise) se place au milieu de l'axe, avec la valence négative (colère, anxiété) située à l'extérieur pour visualiser qu'ici la co-régulation par l'adulte prendra plus de temps qu'en cas d'une émotion à valence positive (joie).

\section{AXE 2: L'intensité (arousal) (faible - moyen -forte) :}

L'activation des circuits neuronaux associés avec le traitement des émotions peut se présenter à différents degrés d'intensité [78, p. 174]. Bien que la culture y soit déjà opérative en conditionnant le jeune membre de la société à favoriser des émotions à intensité forte (sociétés occidentales) ou faibles (sociétés asiatiques) [4, p. 106, 108], on peut distinguer universellement des occasions (a) d'activation du système sympathique causant une fréquence cardiaque élevée et un état d'alerte du système moteur (arousal fort) [65, p. 1051], des situations (b) où on observe une décélération des symptômes décrits comme, par exemple, pendant la provocation d'une réponse de l'interlocuteur adulte [36, p. 59] ou pendant l'imitation de celui-ci comme phase d'orientation, d'apprentissage et d'attente [36, p. 56). Suivant Mauss et al. [49, p. 182-184], on pourrait catégoriser les différents degrés d'intensité d'un évènement émotionnel selon l'activation cardiovasculaire associée à l'émotion observée, en évaluant la joie comme activation forte et la tristesse comme désactivation relative. Cette méthode s'avère peu appropriée ici à cause de l'hypergénéralisation qui en résulterait. Dès lors, nous préférons suivre Yik, Russel et Steiger et al. [79, p.706, Figure 1] et utiliser les 12 secteurs du modèle circomplexe des affects pour classer l'intensité d'un événement émotionnel. Concrètement, et faute d'autres mesures applicables, nous proposons de classifier des manifestations prolongées et très audibles de détresse, colère ou d'enthousiasme comme d'intensité forte, des moments de plaisir, d'irritation ou de tristesse 
partagés avec l'entourage comme d'intensité moyenne et des occasions de contentement ou mécontentement seulement visibles et à durée courte comme d'intensité faible. L'intensité forte sera conséquemment placée à l'extérieur de l'axe, l'intensité faible en position centrique (cf. l'organisation du Geneva Wheel of Emotion [80].

\section{AXE 3: La canalité du traitement co-régulatif de l'adulte (monocanalité - bicanalité - multicanalité) :}

Concernant l'appartenance intrinsèque de la gestion de l'émotion à son expérimentation in situ nous rappelons avec Plantin et Tersis [81, p. 108] que «[d]ans les séquences émotionnelles primaires, l'émotion apparaît, se constitue, est gérée et se dissout éventuellement au fil de l'interaction. » Ce paramètre prend alors en considération qu'un événement émotionnel vécu par le jeune enfant peut être géré par l'adulte en utilisant différents canaux de traitement. Ainsi, dans la pratique quotidienne des éducateurs, on peut observer des occasions où le professionnel opte dans son traitement de l'événement émotionnel pour la monocanalité, p.ex. en raisonnant (canal cognitif) ou en utilisant uniquement le toucher (canal psychomoteur) pour calmer l'enfant qui râle, une fois partis ses parents. D'autres fois, on peut observer une combinaison de plusieurs canaux de traitement, par exemple quand l'éducateur regarde l'enfant avec un sourire encourageant ${ }^{\S \S \S}$ (traitement affectif) en lui faisant un câlin sur la tête (traitement psychomoteur) et en lui expliquant la raison (traitement cognitif) de son comportement. Si cette combinaison intègre deux canaux seulement, nous parlons de bicanalité, si ce sont plus de deux canaux nous parlons de multicanalité. Afin de prévenir des fausses conclusions, nous souhaitons rappeler ici le fait que les interactions citées pour illustrer chaque canal de traitement (i.e. le canal affectif, cognitif ou psychomoteur) ne sont que des exemples. Ainsi, le canal cognitif peut être activé en raisonnant, évidemment, mais une mimique peut également servir pour rendre l'enfant conscient de l'émotion vécue. En ce qui concerne l'ordre des trois états sur l'AXE 3, nous plaçons la monocanalité vers le centre et la multicanalité vers l'extérieur avec la bicanalité au milieu pour représenter l'investissement croissant d'énergie du côté de l'adulte.

\section{AXE 4: La modalité (verbale/non verbale/mixte) de la gestion émotionnelle offerte par l'adulte:}

Cet axe se veut une analyse plus fine du code dont l'adulte se sert pour activer le canal ou les canaux choisi(s) sur l'AXE 3. Le professionnel peut opter, par exemple, pour le code verbal pour inviter l'enfant à une cognitivisation de son ressenti afin de le contrôler, mais il peut également choisir le code non verbal pour aider l'enfant à prendre conscience de l'émotion vécue (p.ex. par la mimique). La même observation vaut pour le canal affectif, car il est également possible de consoler un enfant verbalement qu'à travers la gestuelle ou un toucher réconfortant. Le canal psychomoteur est le seul qui ne peut être activé que de façon non verbale et qui est donc issu d'une corrélation unidirectionnelle. Comme le code non verbal peut se dérouler de façon simultanée pendant que le langage est un médium séquentiel, nous plaçons la modalité non verbale vers le centre, la modalité verbale vers l'extérieur et la modalité mixte au milieu, car l'usage de la gestuelle peut raccourcir l'intervention corégulative de façon significative.

$\S \S$ Concernant le résultat psycho-physiologique positif d'une attitude enthousiaste et optimiste cf. Lundh [82, p. 136]. 


\section{AXE 5: Le degré de familiarité des mots utilisés par l'adulte jugé du point de vue de l'enfant (connus - en train d'être acquis - inconnus) :}

Au cas où l'adulte opte pour une verbalisation (gestion verbale ou mixte), nous étudierons s'il utilise des mots connus, donc familiers à l'enfant ou s'il profite de l'occasion pour le familiariser avec de nouvelles façons de s'exprimer. L'usage des mots familiers aideront à raccourcir l'intervention co-régulative, donc nous la plaçons vers le centre, tandis que l'introduction d'un nouveau vocabulaire demandera un discours explicatif plus long. Le mode " non familier » sera alors placé vers l'extérieur avec les mots en voie d'acquisition figurant au milieu de l'axe.

\section{AXE 6: La sémiotisation des émotions (émotion dite - montrée - étayée) :}

Conforme à la théorie de l'interactionnisme de Kerbrat-Orecchioni [68], cet axe rend compte des différents types de verbalisation d'une émotion. Ainsi, premièrement, le locuteur adulte peut choisir de classifier expressis verbis l'émotion qu'il accompagne chez le jeune enfant (émotion dite), en parlant, par exemple, de sa tristesse, de son chagrin ou de sa joie. Cette stratégie est la moins chronophage, de façon qu'elle sera placée vers le centre. Deuxièmement, le locuteur adulte peut utiliser son intonation et d'autres moyens linguistiques ou «marques langagières [italiques dans l'original] » [75, p. 8] - comme, par exemple, la répétition ou l'emploi des hypocoristiques - pour transmettre l'émotion co-régulée (émotion montrée). Cette catégorie part de l'hypothèse de la cohérence émotionnelle qui « est un cas particulier du principe d'accountability, qui veut que le locuteur rende transparents pour l'interlocuteur ses actes et ses discours afin d'en assurer l'intelligibilité [italiques dans l'original] » [81, p. 108]. Toute émotion montrée est alors traitée comme émotion ressentie, la possibilité d'une émotion feinte étant exclue catégoriquement du modèle. Un soupçon justifié d'incohérence entre émotion dite ou montrée et émotion ressentie du côté de l'adulte devra conséquemment être documenté sur la base des données recueillies. En ce qui concerne le positionnement de ce type de sémiotisation sur l'AXE 6, nous constatons que cette stratégie prendra plus de temps pour être codée que l'émotion dite. Elle se situe alors au milieu de l'AXE 6. Troisièmement, le pédagogue peut légitimer ouvertement - ou pas - l'émotion documentée chez l'enfant (émotion étayée). Nous pouvons constater ce type de sémiotisation quand l'éducateur donne à l'enfant des arguments en faveur de son émotion (légitimation), par exemple en confirmant qu'aujourd'hui papa est sorti vraiment trop vite ou en l'encourageant à vivre son chagrin ou sa colère. La même chose vaut, au contraire, quand le professionnel essaie d'imposer une autre interprétation du ressenti, par exemple en parlant de la fatigue au lieu de la désespération pour ainsi justifier un comportement moins sensible ou le fait d'ignorer complètement la charge émotionnelle de l'enfant (délégitimation). Cette stratégie, qui provoque souvent des contre-arguments de la part de l'enfant, consomme encore plus de temps de façon qu'elle sera placée à la fin extérieure de l'AXE 6.

Les stratégies mixtes (émotion dite/montrée ou montrée/étayée) figureront sur l'AXE 6 au milieu entre les deux catégories combinées à moins que ce ne soit une combinaison entre l'émotion dite et l'émotion étayée. Dans ce cas-là, on travaillera avec un marquage double (voir Figure 6), en choisissant la verticale claire comme trame de fond pour la forme plus proche du centre et un remplissage colorié semi-transparent pour la partie qui dérive du paramètre extérieur. Comme l'émotion montrée et l'émotion étayée demandent également à l'enfant de déduire de l'interaction verbale de l'adulte ce qu'il veut lui faire ressentir, nous y activons le concept de l'inférence introduit par Micheli [83, p. 35] et rappelé par Polo [33, p. 8-9], mais en l'adaptant au monde de la petite enfance. Ainsi, nous proposons de distinguer ici l'inférence affective à faire quand il s'agit d'une émotion montrée et l'inférence cognitive qui se produit quand c'est une émotion étayée qui joue avec les scripts interactionnels associés 
à des situations routinières. De plus, nous pourrions compléter notre analyse de la sémiotisation des émotions en posant la question si les professionnels activent également des stéréotypes phénoménologiques lexicalisés (p.ex. : "Il ne faut pas que tu sanglotes » pour délégitimer le chagrin de l'enfant) ou s'ils transmettent des stéréotypes émotionnels déterminés par la situation, par exemple quand ils réclament la honte de l'enfant qui a mangé tous les morceaux de pêche du bol de fruits pendant le goûter. S'il vaut la peine d'y intégrer encore un autre code ou un neuvième axe dans le diagramme pour visualiser les résultats respectifs dépendra des résultats du terrain.

\section{AXE 7: Le degré de focalisation sur l'enfant (child-centredness) au long de l'intervention régulatrice (focalisation sur l'émotion de l'adulte - focalisation sur l'émotion de l'enfant - focalisation sur l'échange) :}

Sur cet axe nous visualisons si la gestion des émotions vécues par l'enfant pendant un événement émotionnel se concentre sur les émotions enfantines uniquement (child-centred), si, en revanche, on thématise les émotions de l'adulte pour modifier le ressenti de l'enfant (adult-centred) ou si la gestion de l'événement émotionnel essaie de mettre en place un équilibre entre les sentiments de l'enfant impliqué et ceux de son environnement afin de rétablir l'équilibre émotionnel de l'enfant. Il ne s'agit donc pas d'analyser la gestion des dynamiques émotionnelles qui surgissent dans le cadre des interactions entre pairs, mais nous étudions les stratégies de co-régulation appliquées par l'adulte afin d'acheminer la future autorégulation du jeune enfant. Ici, nous plaçons de nouveau les différentes stratégies en ordre croissant du temps et de l'énergie investis dans la co-régulation, en attribuant la place centrale à la focalisation sur l'émotion de l'adulte, la place au milieu à la focalisation sur l'émotion de l'enfant et la position à l'extérieur à la focalisation sur l'échange qui est la stratégie la plus compliquée.

\section{AXE 8: La façon dont l'événement émotionnel se situe (émotion situationnelle - émotion contagionnée par les pairs - émotion induite par un album) :}

En vue de l'importance de la situation [84, p. 43] [85, p.51-52] comme déterminant central de toute sorte de variations linguistique et comportementale [86, p. 77], de l'émotion même [87, p. 16], mais aussi du travail émotionnel (emotion work) [88, p.552] sollicité par des albums et d'autres outils, notre modèle rendra compte du stimulus qui a - apparemment déclenché le ressenti que l'adulte essaie de gérer (ou non). Cette décision n'implique pas que le contexte environnemental fasse partie de l'émotion, c'est-à-dire des changements intérieurs que l'organisme expérimente ou réalise, mais il fait partie de l'épisode émotionnel en s'inscrivant dans la perception mémorisée (ou non) du ressenti vécu de la part de l'individu. Par conséquent, le contexte y sera lui-même sujet à une possible - ou probable transformation à cause du comportement extériorisé ou intériorisé de l'expérimentateur. De même, nous comprenons ici le terme de stimulus dans son sens skinnérien comme " une classe d'événements du monde physique qui partagent la propriété d'influencer les réactions d'un organisme (qu'il s'agisse de les déclencher [...] ou de moduler leur probabilité d'apparition » [89, p. 255]. Par exemple, le fait de voler le doudou à un enfant de deux ans provoquera chez l'enfant sain presque automatiquement une émotion fortement négative - le vol (événement du monde physique) y servant de déclencheur -, tandis que la sortie de la mère ou du père, le matin, augmentera seulement la probabilité d'une réaction émotionnelle négative sans la déclencher nécessairement. Nous nous intéressons à l'influence que la situation exerce sur la complexité du comportement co-régulatif offert par l'adulte, ce qui nous mène à comparer uniquement des événements qui partagent la même valence et le même degré d'arousal chez le jeune enfant. En ce qui concerne le placement des différents types de 
situationalité, nous rappelons ici qu'un événement émotionnel induit par un support médiatique peut se gérer normalement assez vite, alors que l'arousal causé par un événement du monde physique aura une répercussion sur l'organisme du jeune enfant qui demandera un travail émotionnel plus étendu. L'émotion contagionnée par un pair s'y trouvera au milieu, parce qu'elle n'est pas aussi virtuelle que celle d'une figure d'album, ni aussi réelle que celle que l'enfant a expérimentée lui-même.

\section{Le mode de visualisation: l'« octogone des événements émotionnels »}

Une fois déterminés et justifiés ces paramètres afin d'aider les professionnels des structures bilingues-biculturelles à établir un travail émotionnel congruent et interdidactique [7, p. 491], nous devons nous interroger sur la façon dont il serait pertinent d'arranger les axes respectifs. Le défi d'y intégrer, en plus, les stratégies non-verbales [90, p. 326] [91] [92, p. 349] [93] sous forme d'une labellisation verbale, représente une difficulté additionnelle qui est très importante à résoudre. Une solution viable dont nous pourrions nous doter ici, est de choisir un diagramme radar (Figure 1) ou de Kiviat ${ }^{* * * *}$ (Figure 2), parce que ce type de visualisation permet de prendre en compte plusieurs variables indépendantes ou concomitantes.

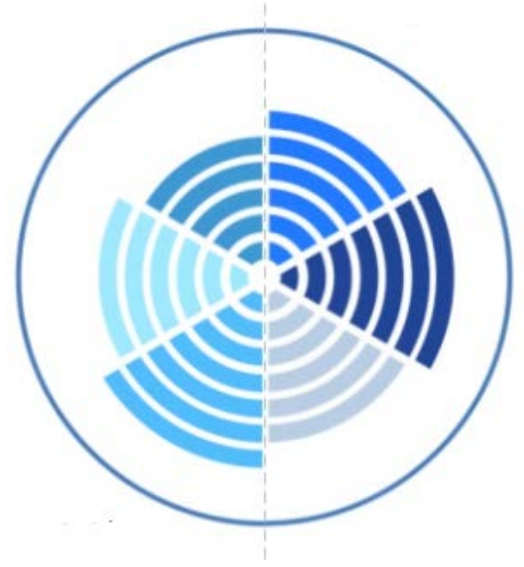

Fig. 1: Diagramme radar

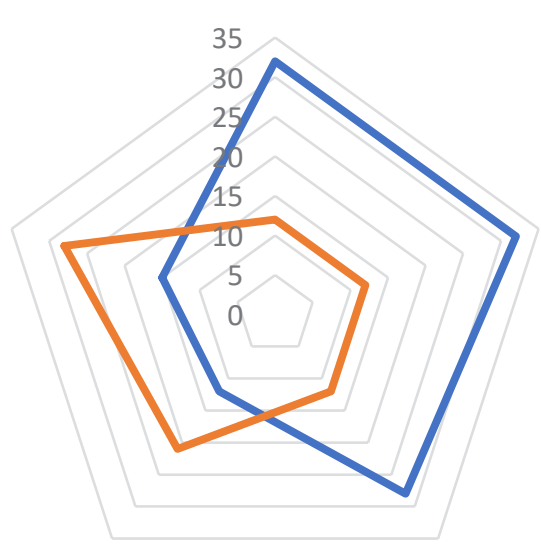

Fig. 2: Diagramme de Kiviat ou d'araignée

Pendant que le diagramme radar présente l'avantage de ne pas suggérer une corrélation intrinsèque entre les points d'extension comparables - comme le sont, par exemple, le cercle intérieur coaxial au moyeu (Figure 3, position a) ou le cercle extérieur (Figure 3, position c) -, il ne sert que pour des cas où les phénomènes à observer se présentent de façon gradée. Comme les phénomènes pris en considération sur les huit axes de notre analyse sont parfois des variables continues (quantitatives) (p.ex. AXE 1 : «Valence » ou AXE 2 : «Intensité »), parfois des variables discrètes catégorielles (qualitatives, sans nécessité d'ordre logique) (p.ex. AXE 3 : «Canalité » ou AXE 6 : «Sémiotisation »), nous donneront la préférence au diagramme de Kiviat. De plus, nous travaillons avec un remplissage semi-transparent de la forme résultant de notre analyse sans que ce remplissage implique une relation d'inclusion

${ }^{* * * *}$ Les diagrammes de Kiviat permettent de visualiser une observation multivariée prenant en compte un nombre élevé de variables sous forme d'une figure géométrique saillante qui facilite l'analyse comparative des situations décomposées [2, p. 617]. 
entre les points plus centriques (ici: a, b) et ceux qui se trouvent plus vers l'extérieur de l'axe (ici: c) (Figure 3). Il vaut alors: $\mathrm{a} \notin \mathrm{b}, \mathrm{c} ; \mathrm{b} \notin \mathrm{c}$.

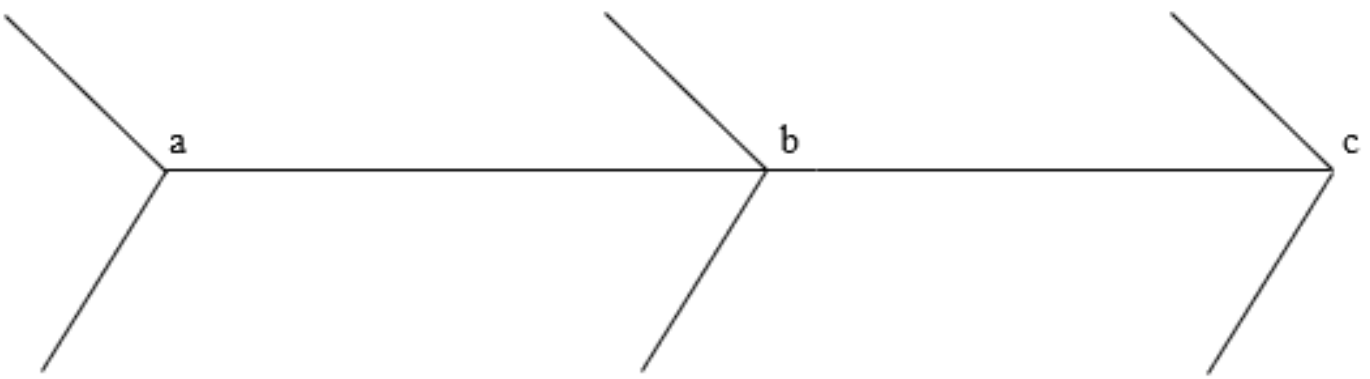

Fig. 3: États d'une caractéristique représentés sur l'axe respectif

Cette solution offre deux avantages : Premièrement, nous pourrons représenter trois états distincts d'une même variable sur le même axe (p.ex. AXE 8: l'émotion induite par la situation, par les pairs ou par un album) ce qui rend le modèle très clair. Deuxièmement, ce modèle nous permettra d'obtenir, quand même, à la fin des analyses des formes n-gonales qu'on pourra superposer afin de visualiser à travers la congruence ou non-congruence géométrique des figures la similitude ou dissimilitude interactionnelles des patrons observés. Pour éviter de possibles malentendus, nous rappelons ici que bien que les états observés sur différents axes puissent trouver leur place à équidistance du centre de la figure (p.ex. en position $\mathrm{b}$ ), ce positionnement analogue n'implique pas forcément que les deux phénomènes soient corrélés. À titre d'exemple d'une vraie corrélation on peut faire référence au lien entre le traitement verbal (position c de l'AXE 4 " modalité ») - plus extensif que le traitement non verbal - et l'émotion étayée ou argumentée (position c de l'AXE 6 "sémiotisation »), phénomène également chronophage. À titre de contre-exemple nous pouvons citer la relation entre le traitement verbal (position c de l'AXE 4 « modalité ») et l'émotion dite (position a de l'AXE 6 « sémiotisation»), la dernière pouvant se limiter in extremis à un mot-phrase (p.ex. "Désolé ! / " Surprise ! »). Les figures géométriques que nous obtiendrons de chaque analyse interactionnelle se voudront alors des instantanés individuels qui seulement après une comparaison quantitative pourront donner lieu à une classification typifiante qui nous informera sur des possibles préférences liées au sexe, à l'âge ou à la biographie langagière de l'acteur professionnel, respectivement à la situation, la structure ou le pays où on gère la structure. Le modèle que nous déduisons de ces réflexions préliminaires s'organisera alors sous forme d'un modèle de Kiviat à huit axes et trois niveaux par axe et nous le nommerons l'« octogone des événements émotionnels » (cf. Figure 4). 


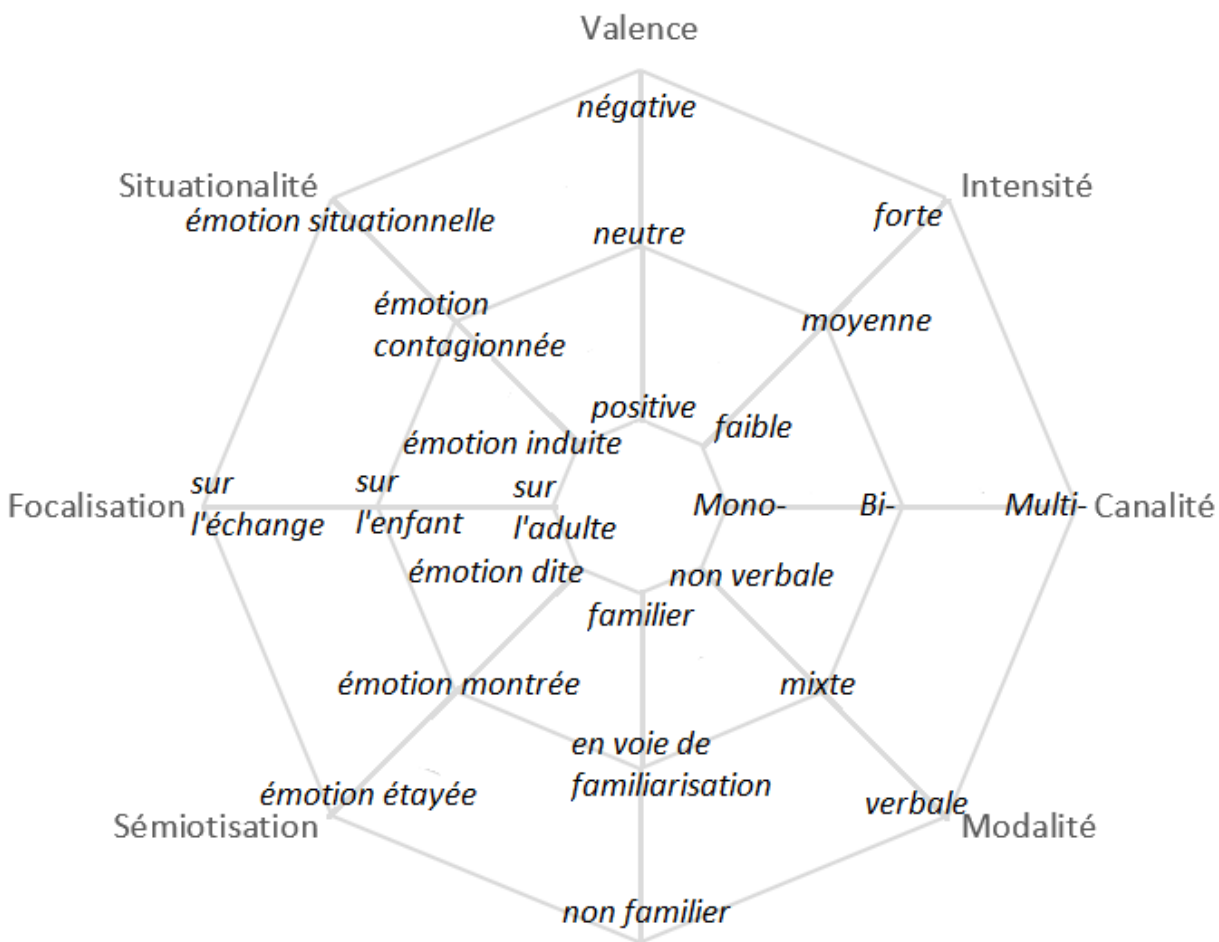

Familiarité

Fig. 4: L'octogone des événements émotionnels (Modèle ciblé aux analyses comparées)

Cette visualisation multi-dimensionnelle des évènements émotionnels et de leur traitement s'avère un point de départ prometteur pour toute étude visant une comparaison de la verbalisation des émotions dans différentes langues car elle s'abstient d'une catégorisation verbale a priori. De plus, en choisissant la forme d'une toile d'araignée, il est possible de conjuguer les valeurs ou degrés d'actualisation sur plusieurs axes en même temps. Ainsi, il sera possible de comparer des événements à forme similaire sans devoir se fonder sur une description uniquement lexicale, qui devrait opter pour l'une des deux langues en jeu. Néanmoins, et avant de commencer à analyser des corpus larges sur la base de l'octogone, nous sommes censée démontrer son applicabilité fiable. Le dernier chapitre de cet article offrira, par conséquent un test d'applicabilité pour évaluer la validité et l'utilité du modèle offert.

\section{Tests d'applicabilité de l'octogone émotionnel dans un contexte bi-plurilingue et bi-pluriculturel}

Pour démontrer si le modèle proposé représente vraiment ce qu'il est censé représenter (critère de validité) et pour tester son utilité dans le champ des études interculturelles, nous l'utiliserons à la suite comme base d'analyse pour six événements émotionnels observés chez quatre éducateurs (dont deux francophones, deux germanophones) travaillant dans des crèches franco-allemandes du Rhin supérieur. Pour assurer la comparabilité des événements analysés au-delà de ces variables extérieures, nous fixons d'abord la valeur sur l'AXE 1 ("Valence ") en optant pour une charge émotionnelle négative et nous standardisons la valeur sur l'AXE 8 ( "Situationalité ») en choisissant une émotion situationnelle, à savoir le chagrin causé par la séparation du parent pendant l'accueil du matin (déclencheur extérieur). 
De plus, nous regardons uniquement des scènes observées pendant la deuxième ou troisième journée du stage immersif pour que les effets du paradoxe de l'observateur soient également comparables.

Ainsi, chaque événement sera transcrit et analysé selon les huit paramètres introduits cidessus et nous ferons figurer les valeurs qui en résulteront sur les axes du modèle. Ce faisant, nous obtiendrons des figures géométriques que nous opposerons l'une à l'autre selon le critère "langue du professionnel» (exemples A et B pour le français, exemples C et D pour l'allemand) et selon le critère «structure » (exemples A et D pour structure 1, exemples B et C pour structure 2). Si la méthode de transformation des observations concrètes en valeurs abstraites est réussie, les formes résultantes des analyses faites par différents chercheurs se ressembleront et aucune difficulté ne se présentera pour interpréter les divergences ou convergences à observer entre les façons différentes de gérer la situation.

\subsection{Application du modèle à deux événements émotionnels gérés par des professionnels francophones}

\section{Exemple A): C1J02.801.941-924.802-901}

Éd. (à l'enfant): Salut! (*) (..) Salut! (*) (Bisou, bisou, bisou. Le prend dans les bras, lui donne des bisous sur la tête, lui fait un câlin sur la tête, lui enlève les cheveux du visage) Ma petite puce. Ça va? T'es en forme?

Parent : $(2 \mathrm{sec})$ Oui. Ça va. Il est (***)...

Éd : Ça marche. (rire) Pas de soucis. Tu dis « au revoir papa »? (vers le père) Vous êtes un peu réservés aujourd'hui? [ $5: 13$ ] Hein ? (vers l'enfant) Dites-moi.

Père : Bonne journée ! (dit « au revoir » avec la main)

Éd. : Merci. Au revoir ! Une bonne journée. « Au revoir, papa », un bisou?

Père : Tant pis ! (lui lance un bisou volant) Bon. Au revoir !

Enfant : Ä-ä-ä-ä-mmm (il se presse contre l'éd. et ne veut plus rien faire)

Éd. (sourit) : Au revoir ! Houp ! (le laisse sauter sur ses bras et le pose doucement par terre à quelques mètres)

\section{Exemple B): C2J02.901.-901.801-901}

(La maman d'un enfant laisse l'enfant et sort vite)

Enfant : Maman ... Maman...Maman ! Maman! (L'enfant reste debout, tourné vers la porte, il serre dans sa main le doudou et les larmes coulent de ses joues et il appelle sa mère crescendo)

Éd.: Nonomo*. (..) Nonomo. (...) Viens ! On va chercher quelque chose pour jouer. Tu veux construire un peu?

Enfant: (Reste immobile, ne regarde pas, fixe la porte avec les yeux.)

Éd.: No-nomo! (..) Écoute, on va chercher quelque chose qui te plaît. Tu veux que je te lise une histoire ? (...) (il s'agenouille)

Enfant: (ne le regarde pas) Maman! (..) Ma-man! (..) Ma-ma:n!

Éd.: Regarde, il ne faut pas que tu sois triste. Nous sommes tous ici pour jouer, pour nous amuser... Regarde tes copains! (montre avec l'index autour d'eux) Ils sont tous ici parce que maman et papa doivent travailler. Mais c'est pas grave. On ne doit pas être triste. Ta maman a dû aller à travailler et elle va revenir le plus vite possible!

Enfant : Non. Elle ne revient pas vite. (..) Maman !

Éd : Alors ... comme tu veux. Tu restes ici et nous, nous allons nous amuser. (se lève et le laisse seul jusqu'à ce qu'il s'approche au coin des livres après un quart d'heure de pleurs désespérés)

*Nonomo représente un prénom masculin à trois syllabes

Pour guider l'interprétation des événements analysés et afin de rendre transparent le passage du texte à la visualisation, nous présenterons d'abord un tableau synoptique (Tableau 1) qui relie les indices trouvés dans le texte avec leur catégorisation respective. Les figures qui en résulteront seront exposées à la suite sous le titre de Figure 5 et Figure 6. 
Tableau 1: Synopse du comportement co-régulatif d'exemple A et B (professionnels francophones)

\begin{tabular}{|c|c|c|}
\hline & Exemple A & Exemple B \\
\hline $\begin{array}{ll}\text { AXE } & 1: \\
\text { Valence } & \end{array}$ & $\begin{array}{l}\text { Négative : } \\
\text { - } \quad \text { pleurs (Ä-ä-ä-ä-mmm) } \\
\text { réaction corporelle (se } \\
\text { presse contre l'éd. et ne } \\
\text { veut plus rien faire) }\end{array}$ & $\begin{array}{l}\text { Négative : } \\
\text { - } \quad \text { pleurs (Maman ... Maman... Maman ! } \\
\text { Maman !) } \\
\text { réaction corporelle (Reste immobile, ne regarde } \\
\text { pas, fixe la porte des yeux.) }\end{array}$ \\
\hline $\begin{array}{ll}\text { AXE } 2: \\
\text { Intensité }\end{array}$ & $\begin{array}{l}\text { Moyenne : } \\
\text { l'enfant montre son } \\
\text { désaccord, mais il se } \\
\text { laisse poser par terre à } \\
\text { quelques mètres (i.e. } \\
\text { très vite) }\end{array}$ & $\begin{array}{l}\text { Forte : } \\
\text { - l'enfant montre son désaccord pendant un quart } \\
\text { d'heure et il défend verbalement son droit d'être } \\
\text { chagriné (Non. Elle ne revient pas vite.) }\end{array}$ \\
\hline $\begin{array}{l}\text { AXE } 3: \\
\text { Canalité }\end{array}$ & $\begin{array}{l}\text { Multicanalité: } \\
\text { - canal psychomoteur: } \\
\text { l'éd. le prend sur les } \\
\text { bras, le laisse sauter } \\
\text { - canal affectif : bisous, } \\
\text { sourire, ma petite puce } \\
\text { canal cognitif: Ça } \\
\text { marche./Pas de soucis. }\end{array}$ & $\begin{array}{l}\text { Monocanalité: } \\
\text { - canal cognitif : l'éd. parle à l'enfant pendant tout } \\
\text { l'événement émotionnel accompagné en lui } \\
\text { proposant à plusieurs reprises des activités } \\
\text { amusantes (Écoute, on va chercher quelque } \\
\text { chose qui te plaît.), en lui rappelant le } \\
\text { comportement plus apprécié des autres enfants } \\
\text { (Regarde tes copains! [...] Ils sont tous ici } \\
\text { parce que maman et papa doivent travailler.) et } \\
\text { en réfutant la légitimité de sa tristesse (Mais } \\
\text { c'est pas grave. On ne doit pas être triste. Ta } \\
\text { maman a dû aller à travailler et elle va revenir } \\
\text { le plus vite possible !) } \\
\text { Note : Le fait de s'agenouiller pourrait être } \\
\text { interprété comme canal affectif, mais on peut } \\
\text { douter de l'effet consolateur au vu de la réaction } \\
\text { de l'enfant et compte-tenu du focus sur le } \\
\text { raisonnement comme stratégie. }\end{array}$ \\
\hline $\begin{array}{l}\text { AXE } 4: \\
\text { Modalité }\end{array}$ & $\begin{array}{l}\text { Mixte : } \\
\text { - } \\
\text { signaux verbaux : Ma } \\
\text { petite puce./Vous êtes } \\
\text { un peu réservés } \\
\text { aujourd'hui?/Tu dis } \\
\text { «aur revoir } \\
\text { papa »?/T'es r en } \\
\text { forme? } \\
\text { signaux non verbaux : } \\
\text { câlins, contact } \\
\text { corporel, sourire }\end{array}$ & $\begin{array}{l}\text { Verbale : } \\
\text { - } \quad \text { signaux verbaux : tout le discours transcrit }\end{array}$ \\
\hline $\begin{array}{l}\text { AXE 5: } \\
\text { Familiarité }\end{array}$ & $\begin{array}{l}\text { Langage familier: } \\
\begin{array}{ll}\text { Cha marche./Pas } & \text { de } \\
\text { soucis/T'es } & \text { en } \\
\text { forme?/Ça va? } & \\
\end{array}\end{array}$ & $\begin{array}{l}\text { Langage familier: } \\
\text { - jouer, plaire, s'amuser, copains, mama, papa, } \\
\text { triste }\end{array}$ \\
\hline $\begin{array}{l}\text { AXE 6: } \\
\text { Sémiotisation }\end{array}$ & $\begin{array}{l}\text { Emotion montrée : Ma petite } \\
\text { puce. T'es en forme? } \\
\text { Émotion étayée : Vous êtes } \\
\text { un peu réservés aujourd'hui ? } \\
(\rightarrow \text { positionnement entre } \\
\text { point b et c) }\end{array}$ & $\begin{array}{l}\text { Émotion dite: triste, s'amuser ( } \rightarrow \text { marque double }) \\
\text { Emotion étayée : } \\
\text { - L'éd. nie le droit à la tristesse et impose la joie } \\
\text { comme émotion socialement attendue. }\end{array}$ \\
\hline $\begin{array}{l}\text { AXE 7: } \\
\text { Focalisation }\end{array}$ & $\begin{array}{l}\text { Focalisation sur l'enfant: } \\
\text { - Les } 5 \text { interactions de } \\
\text { l'éd. se dirigent } \\
\text { presque exclusivement }\end{array}$ & $\begin{array}{l}\text { Focalisation sur l'échange : } \\
\text { - Focus sur l'enfant : tu veux, qui te plaît, que tu } \\
\text { sois triste }\end{array}$ \\
\hline
\end{tabular}




\section{ICODOC 2019}

\begin{tabular}{|c|c|c|c|}
\hline & $\begin{array}{l}\text { vers l'enfant Les } \\
\text { émotions de l'adulte } \\
\text { (Pas de soucis/un peu } \\
\text { réservés) ne sont pas } \\
\text { prises en compte pour } \\
\text { modifier le ressenti de } \\
\text { l'enfant }\end{array}$ & $\begin{array}{l}\text { Focus sur les autres : Nous sommes tous ici } \\
\text { pour jouer, pour nous amuser... Regarde tes } \\
\text { copains !/ Nous, nous allons nous amuser }\end{array}$ \\
\hline $\begin{array}{l}\text { AXE 8: } \\
\text { Situationalité }\end{array}$ & $\begin{array}{c}\text { Émotion situationnelle : } \\
\text { Déclenchée par la la } \\
\text { sortie du parent }\end{array}$ & $\begin{array}{l}\text { Émotion situationnelle : } \\
\text { Déclenchée par la sortie du parent }\end{array}$ \\
\hline
\end{tabular}

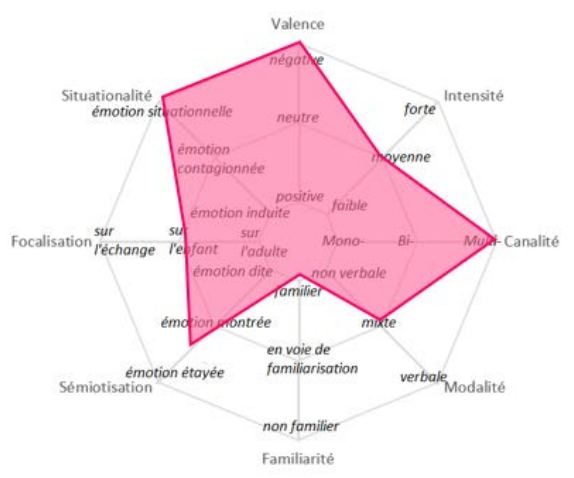

Fig. 5: Co-régulation exemple A (francophone)

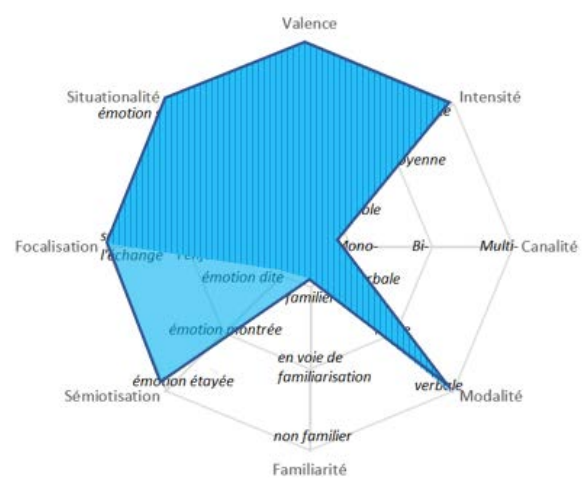

Fig. 6: Corégulation exemple B (francophone)

Si nous profitons maintenant de la possibilité d'une comparaison purement visuelle en superposons les deux figures issues de notre analyse (Figure 7), nous pourrions constater un écart bien évident entre les deux événements émotionnels et leur gestion professionnel par les deux éducateurs francophones observés. 


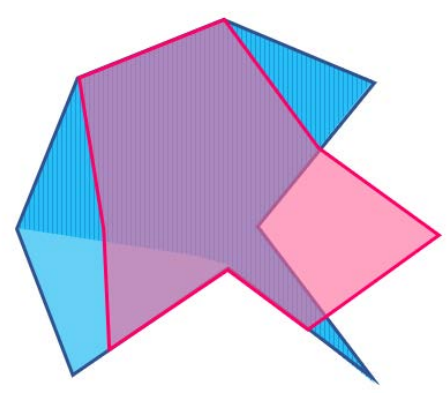

Fig. 7: Comportement co-regulatif francophone : exemple A vs. exemple B

Néanmoins, cette capture momentanée n'informe pas encore sur la nature de la discrépance observée. Ce qu'on peut confirmer sur la base de nos données et analyses est que la gestion par émotion étayée (exemple B) est, en fait, beaucoup plus chronophage (46 mots de la part de l'éd. d'exemple A vs. 104 mots de la part de l'éd. d'exemple B) et que le fait de la combiner avec la monocanalité pourrait encore renforcer cet effet. Si, de plus, une analyse systématique de tous les événements émotionnels enregistrés dans notre corpus révèle une omniprésence de cette diversité comportementale, les visualisations nous aideront à former des sous-corpus avec des situations à profils similaires afin de les soumettre à une analyse plus détaillée des facteurs additionnels qui pourraient être à l'origine de ces divergences (âge/ biographie langagière de l'enfant, éducateur de référence : oui ou non, etc.). Si, au contraire, nous trouvons qu'un des deux profils sera dominant entre les éducateurs francophones, l'hypothèse culturelle tiendra et l'autre profil sera à classer comme cas extrême. Il s'agirait alors d'un profil purement individuel. La troisième variante qui pourrait se donner à la fin de l'analyse quantitative serait l'observation d'une congruence relative entre les figures reflétant le comportement co-régulatif des collègues travaillant dans la même structure. Dans ce caslà, nous devrions supposer l'existence d'une culture interdidactique. De toute façon, avant de considérer le test d'applicabilité comme réussi, nous appliquerons le modèle pour analyser deux autres situations gérées en allemand.

\subsection{Application du modèle à deux événements émotionnels gérés par des professionnels germanophones}

\section{Exemple C) : C4J03.823.942-828.802-902}

Éd.: Aah- Mimini** (salue l'enfant avec une voix de plaisir)

Père : Och Schatziputz (la porte sur les bras et lui essuie les larmes; l'enfant se cramponne fortement à son père) (vers l'éd.) Guten Morgen.

Éd: Guten Morgen.

Père: Na dann machen wir mal halt. Hm ?

Éd: (se lève et s'approche pour être à une distance qui permette de prendre l'enfant des bras du père) Hat se nicht gut geschlafen?

Père : Oh [indéfini ; on ne sait pas si oui ou non]. So, komm. Was magst denn mal spielen? (la dépose par terre avec effort) Schau mal - ganz toll! (montre la tour à blocs LEGO qu'a construit un autre enfant).

Éd.: Ja, da können wir mal zusammen schau'n. (.) Mal sehen was sie mag.

Père: Ja. (parle avec une voix rassurante, très basse, laisse sa main sur le dos de l'enfant)

Enfant (reste debout, regarde vers la salle, les larmes coulent encore sur les joues, mais l'enfant ne pleure plus)

Éd.: So, dann sag mal „Tschüss, Papa!“ Sag mal „Bis später!“

**Mimini représente un prénom féminin à trois syllabes. 


\section{Traduction en français}

Éd.: Aah- Mimini** (salue l'enfant avec une voix de plaisir)

Père : Oh, ben choupinette (il a l'enfant dans les bras et lui essuie les larmes; l'enfant se cramponne fortement à son père) (vers l'éd.) Bonjour.

Éd : Bonjour.

Père: Alors, on y va, hein?

Éd: (se lève et s'approche pour être à une distance qui permette de prendre l'enfant des bras du père) Elle n'a pas bien dormi ?

Père : Oh [indéfini ; on ne sait pas si oui ou non]. Allez, viens, à quoi est-ce que tu voudrais jouer ? (la dépose par terre avec effort) Regarde - c'est super, ça! (montre la tour de blocs Lego qu'a construit un autre enfant).

Éd.: Ah oui, ça on peut regarder ensemble. (.) À voir c'qui la tente.

Père: Oui. (parle avec une voix rassurante, très basse, laisse sa main sur le dos de l'enfant)

Enfant (reste debout, regarde vers la salle, les larmes coulent encore sur les joues, mais l'enfant ne pleure plus)

Éd.: Alors, tu dis « Au-revoir papa! » Dis: « À plus tard! »

**Mimini représente un prénom féminin à trois syllabes.

\section{Exemple D) : C1J02.802.806.901-924.802-901}

Père : Hopp. Au revoir ! ((ferme la porte))

Enfant ((en tapant contre la porte fermée)): Papá! Papá!

Éd. 1: So komm.

Éd. 2: Ah, Mann... (secoue la tête avec des lèvres boudeuses)

Enfant: (crie) Papababa

Éd. 1: Jojojojo. Jojojojo ((intonation descendante, de réconfort; elle le porte dans les bras)) ((les cris deviennent plus forts encore) Wenn man nicht bekommt was man will... gel ?

(à moi) Weischt, der Nomo, wenn er was net kriegt... (continue à pleurer)

Enfant: Babáaaaaaa! Babáaaaa!

Éd. 1: (Elle le porte dans les bras et entre à la salle de bain où elle le réconforte, calme, lui lave le visage, l'essuie, lui essuie les larmes sur les joues, sous le menton) So, jetzt wechseln ma erstmal die Windeln.

\section{Traduction en français:}

Père : Hopp. Au revoir ! ((ferme la porte))

Enfant ((en tapant contre la porte fermée)): Papá! Papá!

Éd. 1: Alors, viens.

Éd. 2: Oh mince... (secoue la tête avec des lèvres boudeuses)

Enfant: (crie) Papababa

Éd. 1: Oh-la-la-la-la. Oh-la-la-la-la. ((intonation descendante, de réconfort; elle le porte dans les bras)) ((les cris deviennent plus forts encore) Si on n'a pas ce qu'on veut... hein ?

(à moi) T'sais, Nomo, quand on ne lui donne pas ce qu'il veut... (continue à pleurer)

Enfant: Babáaaaaaa! Babáaaaa!

Éd. 1: (Elle le porte dans les bras et entre à la salle de bain où elle le réconforte, calme, lui lave le visage, l'essuie, lui essuie les larmes sur les joues, sous le menton) Alors, on va changer d'abord.la couche

Nous y procéderons de la même manière qu'à l'étape précédente en présentant d'abord un tableau synoptique qui rend transparent notre travail d'analyse.

Tableau 2: Synopse du comportement co-régulatif d'exemple C et D (professionnels germanophones)

\begin{tabular}{|c|c|c|}
\hline & Exemple C & Exemple D \\
\hline $\begin{array}{ll}\text { AXE } & 1: \\
\text { Valence } & \end{array}$ & $\begin{array}{l}\text { Négative : } \\
\text { - larmes } \\
\text { réaction corporelle (se } \\
\text { cramponne fortement à } \\
\text { son père) }\end{array}$ & $\begin{array}{l}\text { Négative : } \\
\text { - pleurs en crescendo } \\
\text { - réaction corporelle (tape contre la } \\
\text { porte) }\end{array}$ \\
\hline $\begin{array}{ll}\text { AXE } 2: \\
\text { Intensité }\end{array}$ & $\begin{array}{l}\text { Moyenne: } \\
\text { - l'enfant montre son } \\
\text { désaccord, mais il se laisse } \\
\text { poser par terre et arrête de } \\
\text { pleurer bien qu'il reste } \\
\text { vigilant (debout) }\end{array}$ & $\begin{array}{l}\text { Forte : } \\
\text { - l'enfant montre son désaccord de } \\
\text { façon hautement audible et visible } \\
\text { (râle, tape contre la porte, pleure) }\end{array}$ \\
\hline
\end{tabular}




\begin{tabular}{|c|c|c|}
\hline $\begin{array}{l}\text { AXE 3: } \\
\text { Canalité }\end{array}$ & $\begin{array}{l}\text { Bicanalité: } \\
\text { - canal affectif: voix de } \\
\text { plaisir } \\
\text { canal cognitif : demande si } \\
\text { l'enfant a eu une mauvaise } \\
\text { nuit (Hat se nicht gut } \\
\text { geschlafen ?//trad. : Elle } \\
\text { n'a pas bien dormi ?) et } \\
\text { propose de chercher une } \\
\text { activité qui lui plaise } \\
\text { Note : L'éd. se rapproche } \\
\text { pour préparer un possible } \\
\text { passage réconfortant de } \\
\text { bras en bras (canal } \\
\text { psychomoteur ciblé), mais } \\
\text { le père ignore le geste } \\
\text { décidemment, le vidant } \\
\text { ainsi de son sens. }\end{array}$ & $\begin{array}{l}\text { Multicanalité: } \\
\text { - canal psychomoteur: l'éd. le prend } \\
\text { sur les bras, lui lave le visage, } \\
\text { l'essuie, lui essuie les larmes sur les } \\
\text { joues, sous le menton) } \\
\text { canal affectif: } \\
\text { descendante, de réconfort } \\
\text { canal cognitif: Wenn man nicht } \\
\text { bekommt was man will... gel ?\|t trad. : } \\
\text { Si on n'a pas ce qu'on veut...hein? }\end{array}$ \\
\hline $\begin{array}{l}\text { AXE 4: } \\
\text { Modalité }\end{array}$ & $\begin{array}{l}\text { Verbale: } \\
\text { - } \text { signaux verbaux : } \\
\text { salutation, propos attractif, } \\
\text { lui donne des mots pour } \\
\text { gérer la séparation }\end{array}$ & $\begin{array}{ll}\text { Mixte : } \\
\text { - } & \text { signaux verbaux : interjections } \\
& \text { (Jojojojo. Jojojojo), dialogue directe } \\
\text { - } & \text { signaux non verbaux: câlins, soin, le } \\
& \text { prendre sur les bras }\end{array}$ \\
\hline $\begin{array}{l}\text { AXE 5: } \\
\text { Familiarité }\end{array}$ & 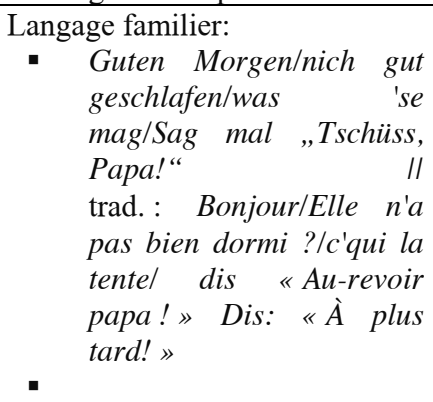 & $\begin{array}{l}\text { Langage familier: } \\
\text { - So komm./Wenn man nich kriegt, was } \\
\text { man will./ Windeln wechseln } \| \text { trad. : } \\
\text { Alors, viens. Si on n'a pas ce qu'on } \\
\text { veut... hein?/changer la couche }\end{array}$ \\
\hline $\begin{array}{l}\text { AXE 6: } \\
\text { Sémiotisation }\end{array}$ & $\begin{array}{l}\text { Emotion étayée : } \\
\text { - l'éd. propose la fatigue } \\
\text { (nich gut geschlafen } \| \text { trad. } \\
\text { n'a pas bien dormi) } \\
\text { comme raison du chagrin } \\
\text { pour sauver la face du } \\
\text { parent }\end{array}$ & $\begin{array}{l}\text { Émotion montrée : Jojojojo. Jojojojo } \| \\
\text { trad. : Oh-la-la-la-la. Oh-la-la-la-la. } \\
\text { Emotion étayée: } \\
\text { - Wenn man nich kriegt, was man will. } \\
\text { II trad.: Si on n'a pas ce qu'on veut } \\
\text { les pleurs sont interprétés comme } \\
\text { symptôme de colère } \\
\text { So, jetzt wechseln ma erstmal die } \\
\text { Windeln. II trad. : Alors, on va } \\
\text { changer d'abord la couche. } \\
\text { L'éd. présente une interprétation } \\
\text { alternative (la couche pleine) pour } \\
\text { ainsi affirmer son autorité et droit de } \\
\text { s'enlever l'enfant profondément } \\
\text { chagriné } \\
(\rightarrow \text { positionnement entre point b et c) }\end{array}$ \\
\hline $\begin{array}{l}\text { AXE } 7: \\
\text { Focalisation }\end{array}$ & $\begin{array}{l}\text { Focalisation sur l'enfant: } \\
\text { - Les interactions verbales } \\
\text { de l'éd. se concentrent } \\
\text { uniquement sur l'enfant. }\end{array}$ & $\begin{array}{l}\text { Focalisation sur l'enfant : } \\
\text { - L'éd. parle tout le temps avec l'enfant } \\
\text { et s'occupe de lui }\end{array}$ \\
\hline $\begin{array}{l}\text { AXE 8: } \\
\text { Situationalité }\end{array}$ & $\begin{array}{l}\text { Émotion situationnelle : } \\
\text { - Déclenchée par la sortie du } \\
\text { parent }\end{array}$ & $\begin{array}{l}\text { Émotion situationnelle : } \\
\text { - Déclenchée par la sortie du parent }\end{array}$ \\
\hline
\end{tabular}




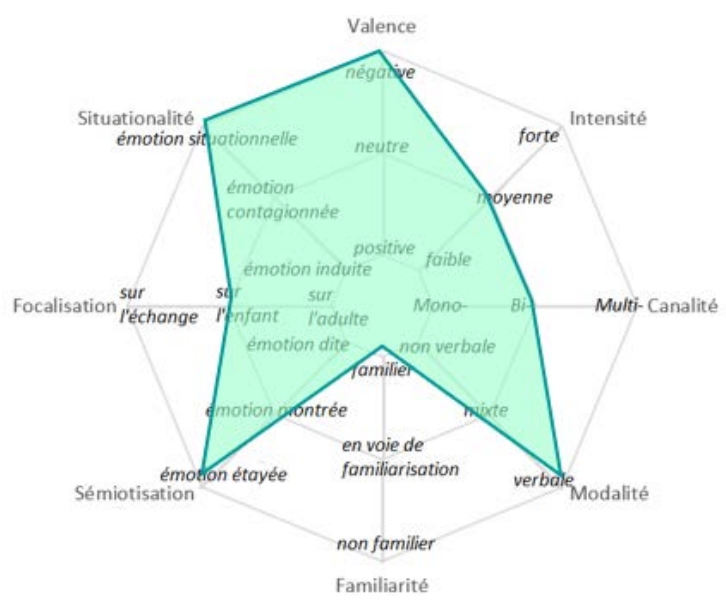

Fig. 8: Co-régulation exemple D (germanophone)

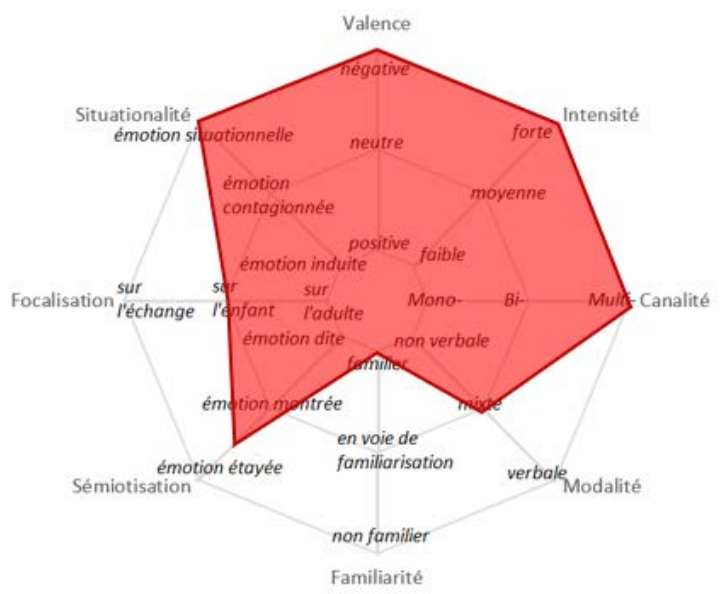

Fig. 9: Co-régulation exemple $\mathrm{C}$ (germanophone)

L'analyse verbalisée des deux transcriptions démontre la difficulté de classer les différentes interactions composant un seul événement émotionnel co-régulé par l'adulte et le tableau, bien que très comprimé dans sa présentation, n'aide pas précisément à se faire vite une idée claire sur les stratégies partagées par les deux professionnels germanophones ici étudiés. Si nous recourons, au contraire, à la visualisation en superposant les deux formes résultantes des exemples $\mathrm{C}$ et $\mathrm{D}$ (Figure 10), il est beaucoup plus facile de voir qu'au-delà de l'intensité de l'émotion enfantine comme variable indépendante du professionnel, il y a trois paramètres qui causent la divergence entre le comportement de l'éducateur $\mathrm{C}$ et $\mathrm{D}$ : premièrement, le choix des canaux (bicanalité dans le cas de $\mathrm{C}$ vs. multicanalité dans le cas de D) comme variable contrôlée par l'adulte, deuxièmement l'usage exclusif de la langue comme moyen de co-régulation dans le cas d'exemple $\mathrm{C}$ et, troisièmement, la récurrence plus (exemple C) ou moins (exemple D) exclusive à la stratégie de l'émotion étayée quand le chagrin enfantin est ignoré sous prétexte d'autres interprétations de la manifestation émotionnelle négative (colère, fatigue, malaise corporel à cause d'une couche pleine). 


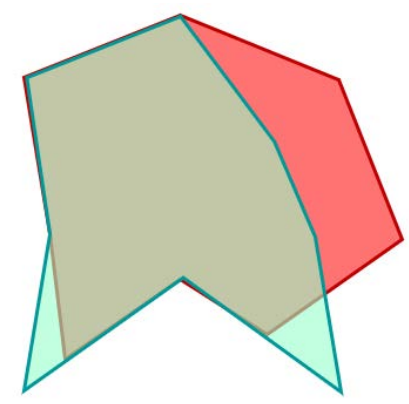

Fig. 10: Comportement co-regulatif germanophone: exemple C vs. exemple D

De même que dans le cas des professionnels francophones, l'octogone des événements émotionnels nous aide à découvrir des profils comportementaux divergents entre des locuteurs natifs de la langue allemande. Cela implique que l'hypothèse culturelle ne peut pas se confirmer simplement, à moins qu'une révision ample de tous les exemples de notre corpus puisse porter la preuve d'une préférence significative pour l'un des deux profils. La seule conclusion préliminaire que nous puissions présenter, ici basée sur les quatre exemples, est celle d'une diversité interindividuelle et inter-situationnelle remarquable ce qui signifie que le modèle sert son but.

\subsection{Application du modèle à deux événements émotionnels gérés par des collègues de la même structure}

Une autre hypothèse que nous proposons de tester ici afin de montrer la validité et l'utilité de l'octogone émotionnel pour des recherches interculturelles est celle de la possibilité de visualiser des congruences comportementales au sein d'une structure. Comme les professionnels $\mathrm{B}$ et $\mathrm{C}$ ne travaillent pas dans la même structure, nous ne pouvons offrir ici qu'une comparaison entre le comportement observé dans les exemples A et D, mais ceci avec un résultat impressionnant: les profils des éducateurs observés dans les cas A et D se ressemblent complètement à l'exception de la moindre intensité de l'émotion gérée dans le cas A. En vue des difficultés et dysfonctionnements d'origine linguistique et culturelle ${ }^{\dagger \dagger \dagger \dagger}$ à prévenir dans des équipes binationales, une telle constellation entre collègues s'avère très prometteuse pour un travail congruent et elle peut s'interpréter comme indice d'une culture pédagogique partagée (i.e. d'interculturalité).

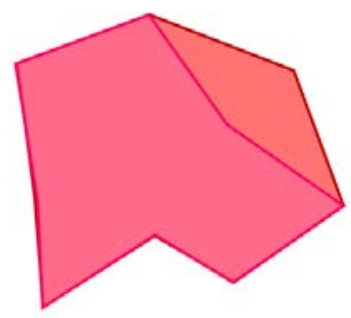

Fig. 11: Comparaison des comportements co-régulatifs de deux collègues travaillant dans la même structures (cas A vs. cas D)

iti† Nous y adoptons le concept des Dysfonctionnements d'Origine Linguistique et Culturelle (DOLC) défini et introduit dans le discours scientifique par J. Breugnot lors de différentes conférences, entre autres au Colloque Xalapa, 15-17 novembre 2017. 
D'un autre côté, on pourrait utiliser notre modèle comme outil en ressources humaines en évaluant les habitudes co-régulatives des candidats à un poste libre dans le cadre d'un stage probatoire. Si on a déjà opté pour analyser les habitudes co-régulatives de ses employés afin d'identifier le profil comportemental de la crèche, il sera possible de le comparer avec les habitudes des futures collègues afin d'identifier des combinaisons harmonieuses ou plutôt conflictuelles. À titre d'exemple pour ce deuxième scénario, nous pourrions citer une collaboration fictive entre les professionnels des exemples B et C (Figure 12).

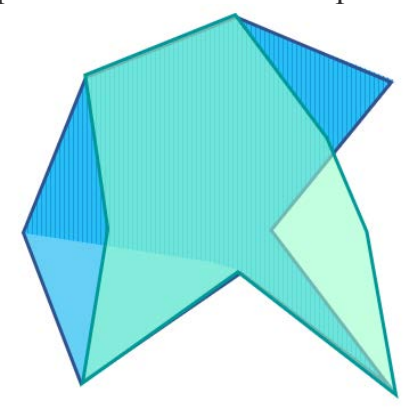

Fig. 12: Comparaison des comportements co-régulatifs de deux futurs collègues à profil divergent (cas B vs. cas C)

Néanmoins, et même si on a déjà une équipe composée des professionnels à profils très divergents, l'octogone peut servir à la direction d'instrument de gestion et de médiation entre les employés, parce qu'il permet de rendre visible et d'objectiver les différences sous-jacentes sans les évaluer grâce à la multi-dimensionalité du modèle. Ainsi, pendant qu'un diagramme à orientation verticale et horizontale invitera toujours à interpréter l'axe des ordonnées comme représentation d'une performance plus haute ou plus basse, la figure n-gonale irrégulière se présente comme plus neutre et plus individualisée ce qui prépare le terrain pour un échange respectueux sur les préférences et particularités de chacun et chacune. Le fait que les figures reflètent chaque événement de façon fidèle et claire, indépendamment des mots et de la langue utilisée, augmentera, de plus, l'acceptabilité du modèle pour des équipes biplurilingues et bi-pluriculturelles parce que la représentation n'impose aucune langue comme plus importante que l'autre. Ces caractéristiques du modèle octogonal portent preuve de la validité et de l'utilité de notre propos pour des analyses interculturelles ainsi que pour les acteurs du terrain.

\subsection{Application du modèle à deux événements émotionnels gérés par code- switching : test d'interdidacticité réussie}

Pour terminer cette introduction d'un nouvel outil d'analyse interactionnelle et interculturelle, nous souhaitons démontrer sa plus-value pour la recherche en présentant encore un exemple qui s'intéresse au cas des professionnels bi-plurilingues actifs, à savoir des professionnels déjà observés dans les exemples $\mathrm{A}(\rightarrow \mathrm{E})$ et $\mathrm{D}(\rightarrow \mathrm{F})$. 


\section{Exemple E): C1J02.801.941-825.802-901}

(Accueil du matin)

Éd. (vers l'enfant) : Allez, ma petite, c'est tout bien ? (vers le père) Aujourd'hui elle vient en bras ? Ce sont des très rares fois qu'on a peine à la recevoir...

Père : Oui. Elle a eu de la douleur de l'oreille. Aujourd'hui c'est mieux. Mais hier elle m'a tout montré

Éd. : Mon Dieu ! Tu vas me montrer ton oreille, hein ? Tu as mal à l'oreille, toi ? Tu veux qu'on la couvre un peu? (la prend sur les bras et la fait s'appuyer avec la tête contre son épaule pour chauffer l'oreille)

[va-et-vient de 10 tours entre le père et l'éd. jusqu'à ce que l'enfant passe de bras en bras; l'éd. lui donne des bisous sur la tête et lui fait des câlins]

Père : Bonne journée !

Enfant : Ääääääääääh äääääääääh (pleure à chaudes larmes)

Éd. : T'as mal à l'oreille?

Enfant (en pleurant) : Jaaaaaa!

Éd. : Hein?

Enfant : Jaaaaaa! [trad.: Ouai:s!]

Éd. : Là, ça fait mal, oui ? Alors on pose la tête un tout petit peu (s'assoit sur une chaise, prends l'enfant sur ses genoux et la fait appuyer son oreille douloureuse contre son épaule pour la chauffer) Ouiiiiii. Ohhhhhh. Mmmmmh (elle fait des petits sons calmants, lui donne des bisous sur la tête, lui caresse la joue) (3 sec.) Hast Du Ohrenschmerzen? [trad. : Tu as mal à l'oreille ?]

\section{Exemple F): C3J03.802-834.814-901}

(Regroupement du matin; l'éd. est déjà installée devant le grand groupe qui s'est installé sous forme de semicercle devant elle; l'enfant est assis à côté de l'éd. et se presse contre elle)

Enfant: Ich hab da Aua, bitte! (montre une toute petite blessure)

[trad.: Là, j'ai un petit bobo, s'il-te-plaît.]

Éd.: Immer wieder aua, hein? Immer ein bobo, ohje.

[trad.: Toujours un bobo, hein ? Mon Dieu, toujours un bobo.]

(caresse brièvement l'intérieur de la main avec une mine de pitié) So. (se redresse) Was sollen wir denn machen?

[Alors, qu'est-ce que vous voulez faire?]

Enfant: Ich hab da wieder ein machen.

[J'y ai encore un bo] [Note: Ici le verbe machen " faire » semble être l'abréviation du verbe phraséologique Aua-machen "faire du mal/causer une douleur». C'est pourquoi je l'ai traduit également par une abbréviation : *bo de bobo. - L'enfant est en train de devenir trilingue; il a commencé à apprendre l'allemand à la crèche..]

(Cette fois-ci, l'éducatrice l'ignore)

Éd. (vers le grand groupe) : Sollen wir das mit den Fingerchen machen?

[Voulez-vous qu'on fasse le jeu avec les petits doigts ?]

Dans les cas $\mathrm{E}$ et $\mathrm{F}$ nous analysons de nouveau des événements négatifs (AXE 1 : "valence négative ") gérés par les deux professionnels travaillant dans la même structure, mais cette fois-ci à l'occasion d'une douleur (AXE 8: "émotion situationnelle "). Si nous regardons les transcriptions des deux scènes à durée et intensité émotionnelle tout à fait différentes (AXE 2: «intensité forte» dans l'exemple E vs. «intensité faible» dans l'exemple F), nous serions tentés de juger le comportement co-régulatif du professionnel dans l'exemple E comme beaucoup plus engagé et complexe que celui de la personne observée dans l'exemple $\mathrm{F}$. Si, au contraire, nous procédons à la microanalyse interactionnelle qui se présentera ci-dessous sous forme de tableau synoptique (Figure 3 ) et si nous visualisons après les résultats à l'aide du diagramme Kiviat proposé, la réalité se présente déjà différemment (Figure 13 et 14).

Tableau 3: Synopse du comportement co-régulatif d'exemple E et F (code-switching vers l'allemand vs. vers le français)

\begin{tabular}{|c|c|c|}
\hline & Exemple E & Exemple F \\
\hline $\begin{array}{ll}\text { AXE } 1: \\
\text { Valence }\end{array}$ & $\begin{array}{l}\text { Négative : } \\
\text { - larmes ininterrompues }\end{array}$ & $\begin{array}{l}\text { Négative : } \\
\text { - l'enfant montre une blessure et demande } \\
\text { gu'on la soigne }\end{array}$ \\
\hline
\end{tabular}




\begin{tabular}{|c|c|c|}
\hline & $\begin{array}{l}\text { - } \text { réaction corporelle (il } \\
\text { prend } 10 \text { tours jusqu'à ce } \\
\text { que l'enfant passe de } \\
\text { bras en bras) }\end{array}$ & $\begin{array}{l}\text { - réaction corporelle: l'enfant se presse } \\
\text { contre l'éd. }\end{array}$ \\
\hline $\begin{array}{ll}\text { AXE } 2: \\
\text { Intensité }\end{array}$ & $\begin{array}{l}\text { Forte: } \\
\text { - l'enfant a des douleurs } \\
\text { d'oreille et ne veut pas se } \\
\text { séparer de son parent; } \\
\text { les larmes continuent et } \\
\text { reviennent pendant un } \\
\text { temps prolongé }\end{array}$ & $\begin{array}{l}\text { Faible: } \\
\text { - l'enfant demande l'attention de l'éd., mais } \\
\text { accepte le commencement du } \\
\text { regroupement du matin sans signaux } \\
\text { négatifs }\end{array}$ \\
\hline $\begin{array}{ll}\text { AXE } 3: \\
\text { Canalité }\end{array}$ & $\begin{array}{l}\text { Multicanalité: } \\
\text { canal affectif: voix et } \\
\text { regards de pitié, ma } \\
\text { petite/Mon Dieu! } \\
\text { canal cognitif : l'éd. } \\
\text { demande à l'enfant l'état } \\
\text { des douleurs (Tu vas me } \\
\text { montrer ton oreille, } \\
\text { hein? Tu as mal à } \\
\text { l'oreille, toi ? Là, ça fait } \\
\text { mal, oui ?) et l'éd. } \\
\text { propose des mesures qui } \\
\text { améliorent la douleur } \\
\text { (Tu veux qu'on la couvre } \\
\text { un peu? ?/on pose la tête } \\
\text { un tout petit peu?) } \\
\text { canal psychomoteur : } \\
\text { l'éd. lui donne des bisous } \\
\text { sur le tête et lui fait des } \\
\text { câlins, lui caresse la } \\
\text { joue, appuye l'oreille } \\
\text { contre son épaule, le } \\
\text { couvre de son écharpe }\end{array}$ & $\begin{array}{l}\text { Multicanalité: } \\
\text { - canal psychomoteur: l'éd. caresse } \\
\text { brièvement l'intérieur de la main } \\
\text { canal affectif: intonation descendante, de } \\
\text { réconfort, ohje } \\
\text { canal cognitif: l'éd. verbalise qu'elle a } \\
\text { compris pourquoi l'enfant est si collant } \\
\text { (Immer wieder aua, hein? Immer ein } \\
\text { bobo) }\end{array}$ \\
\hline $\begin{array}{ll}\text { AXE } 4: \\
\text { Modalité }\end{array}$ & $\begin{array}{l}\text { Mixte : } \\
\text { - } \text { signaux verbaux : ma } \\
\text { petite/Mon Dieu !/ Hast } \\
\text { Du } \\
\text { Ohrenschmerzen?(code- } \\
\text { Switching) } \\
\text { signaux non verbaux: } \\
\text { câlins, porter sur les bras }\end{array}$ & $\begin{array}{l}\text { Mixte : } \\
\text { - } \quad \text { signaux verbaux : interjection (ohje), } \\
\text { demande si elle a bien identifié la cause du } \\
\text { chagrin: Immer wieder aua, hein? Immer } \\
\text { ein bobo (code-switching) } \\
\text { signaux non verbaux: câlins, soin, } \\
\text { s'incliner vers la main blessée }\end{array}$ \\
\hline $\begin{array}{l}\text { AXE 5: } \\
\text { Familiarité }\end{array}$ & $\begin{array}{l}\text { Langage familier: } \\
\text { Allez, ma petite, c'est } \\
\text { tout bien ?/ T'as mal à } \\
\text { l'oreille? }\end{array}$ & $\begin{array}{l}\text { Langage familier: } \\
\text { - } \quad \text { wieder aua, ein bobo }\end{array}$ \\
\hline $\begin{array}{l}\text { AXE 6: } \\
\text { Sémiotisation }\end{array}$ & $\begin{array}{l}\text { Emotion montrée : } \\
\text { - l'éd. démontre sa pitié à } \\
\text { travers la voix, les } \\
\text { regards, les gestes, les } \\
\text { interjections } \quad \text { (Mon } \\
\text { Dieu!) }\end{array}$ & $\begin{array}{l}\text { Émotion montrée : ohje, aua, bobo } \\
\text { Emotion étayée : } \\
\text { - L'éd. souligne que l'enfant a tendance à se } \\
\text { préoccuper pour des petites blessures } \\
\text { (vraies ou imaginées (immer } \\
\text { wieder/wieder = "toujours/ de } \\
\text { nouveau ") } \\
(\rightarrow \text { positionnement entre point b et c) }\end{array}$ \\
\hline $\begin{array}{l}\text { AXE } 7: \\
\text { Focalisation }\end{array}$ & Focalisation sur l'enfant : & $\begin{array}{l}\text { Focalisation sur l'enfant: } \\
\text { - L'éd. ne verbalise que le problème de } \\
\text { l'enfant }\end{array}$ \\
\hline
\end{tabular}




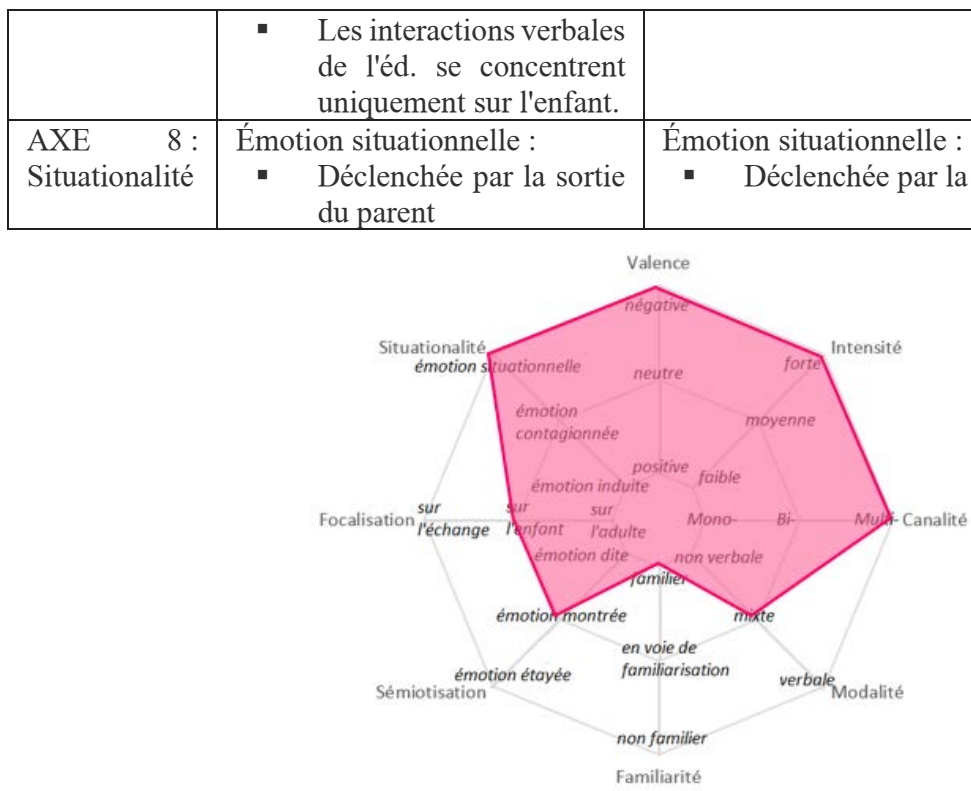

Fig. 13: Co-régulation exemple E (français-bilingue)

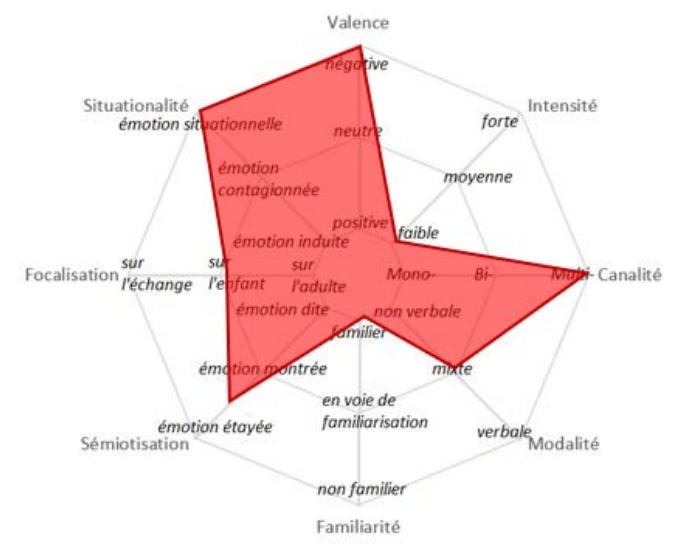

Fig. 14: Co-régulation exemple F (allemand-bilingue)

Bien que ces visualisations laissent déjà reconnaître certaines similitudes, c'est le test de superposition (Figure 15) qui nous aide le plus à formuler un jugement correct concernant les habitudes co-régulatives des deux impliqués.

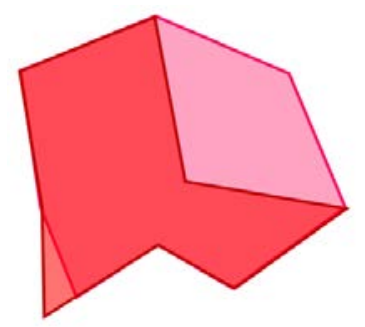

Fig. 15: Comportement co-regulatif bilingue avec code-switching: exemple E vs. exemple F 
Comme nous apprend la Figure 15, l'écart le plus grand entre les deux événements émotionnels analysés découle de l'intensité divergente de l'émotion ressentie par les deux enfants (AXE 2) alors que la différence comportementale entre l'émotion uniquement montrée (exemple E) et le mélange entre émotion montrée et étayée (exemple F) est minime. $\mathrm{Au}$ lieu de nous laisser tromper par l'importance objective de l'événement à gérer, par la longueur de la scène transcrite ou par la langue différente - trois facteurs qui parleraient en faveur d'une interprétation divergente du comportement des deux professionnels -, le modèle ici proposé nous fournit une méthode d'analyse plus précise et plus objective. De même, au lieu de réduire la constatation d'une similitude comportementale entre professionnel francophone (exemples A, E) et professionnel germanophone (D, F) au fait de pratiquer le code-switching pour sécuriser l'enfant dans sa langue dominante, l'octogone ici proposé et testé nous donne les clés pour argumenter en faveur d'un habitus professionnel partagé. Cette observation se voit, de plus, confirmée par une autre comparaison croisée qui opposera la gestion des deux événements émotionnels à intensité forte (exemple D et E) (Figure 16) et les deux événements à intensité faible (exemple F) et moyenne (exemple A) (Figure 17).

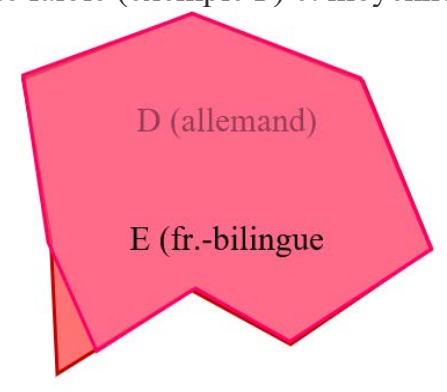

Fig. 16: Comparaison du comportement co-régulatif à l'occasion d'une émotion forte (exemple D vs. E)

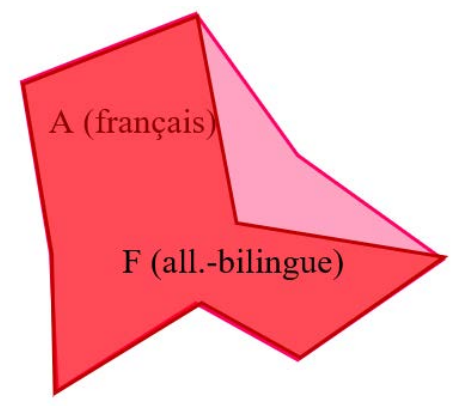

Fig. 17: Comparaison du comportement co-régulatif à l'occasion d'une émotion moins forte (exemple A vs. F)

Les figures 16 et 17 démontrent que le comportement des deux professionnels est congruent entre eux - quelle que soit la langue parlée - et dans le cas des émotions moins intensives (Figure 17) les adultes procèdent même de façon identique (axes 3-7). Cette congruence ne peut se constater ici que pour des événements émotionnels à valence négative (chagrin, douleur) et de façon très préliminaire (sur la base de 4 exemples), mais la méthode d'analyse comparative appliquée s'avère capable d'identifier les congruences et incongruences interindividuelles que nous cherchons pour contribuer à la sensibilisation et médiation interculturelle en équipe bilingue/biculturelle. 


\section{Limitations et perspectives}

Le fait de vouloir utiliser un modèle pour pouvoir attribuer des étiquettes assez simplistes à des interactions hautement complexes s'avère toujours un défi et l'ambigüité ou contestabilité des classifications faites est évidemment la faiblesse majeure de l'octogone des événements émotionnels. Néanmoins, si nous rappelons que le cerveau humain a besoin de simplifications et de patrons abstraits pour pouvoir vivre avec le chaos qui l'entoure, nous pouvons accepter avec E. Morin [94] qu'il n'y a pas de "vérité scientifique» absolue. En revanche, nous pouvons nous contenter avec la fiabilité inter-évaluateur (inter-rater reliability) en soumettant chaque événement émotionnel à une analyse double ou triple de la main de différents chercheurs. L'objectivité qui en découlera nous permettra de recommander le modèle ici proposé pour des recherches interculturelles afin d'en apprendre davantage sur les cadres de référence qui déterminent le travail et l'accueil dans les crèches bi-plurilingues/bipluriculturelles. Le savoir que nous serons capable de construire de cette façon, informera la future construction des curriculums pour la petite enfance et, plus spécifiquement, des projets pédagogiques des structures bilingues/biculturels.

En ce qui concerne l'applicabilité du nouvel outil sur le terrain, celui-ci se recommande surtout dans trois secteurs : la gestion des ressources humaines (cf. 3.3, Figure 12), la médiation interculturelle au sein d'une équipe et la formation continue du personnel. Dans le cadre d'un recrutement de futures collègues, l'octogone des événements émotionnels peut s'utiliser pour identifier des personnes à comportement co-régulatif harmonieux ou complémentaire afin de créer des équipes qui fonctionnent. Si entre les employés qui gèrent un groupe ensemble, on découvre, en revanche, des profils très divergents ou possiblement conflictuels, ce type d'analyses peut servir de base pour mieux planifier les créneaux de travail. Ainsi, on pourrait, par exemple, diminuer la fréquence des escalades émotionnelles le matin en n'y employant l'éducateur dont la force est le travail cognitif avec les enfants plus âgés ou, à l'inverse, le collègue travaillant préférablement sur le canal affectif et psychomoteur pourrait être exempté du service dans les ateliers d'expérimentation. Compte tenu de l'obligation pour les structures d'accompagner leurs employés dans le processus de lifelong learning, l'octogone peut servir d'instrument dans le cadre d'un suivi régulier afin de découvrir de possibles difficultés ou besoins de formation continue. Compte-tenu du fait qu'un comportement non congruent de la part de l'adulte (non congruence intra-individuelle) pourra mener à un attachement insécurisant ou ambivalent du côté de l'enfant, un tel monitoring nous paraît d'autant plus utile. Mais aussi l'identification des professionnels très congruents dans leur comportement co-régulatif peut aider la direction d'une crèche à prendre des décisions appropriées : ainsi, le choix de l'éducateur de référence pour les enfants ayant besoin de plus de sécurisation sera plus facile si on connait les profils comportementaux de ses employés et les stagiaires profiteront également d'un formateur congruent comme le sont les deux professionnels que nous avons analysés dans les exemples A, D, E et F (Figure 18).
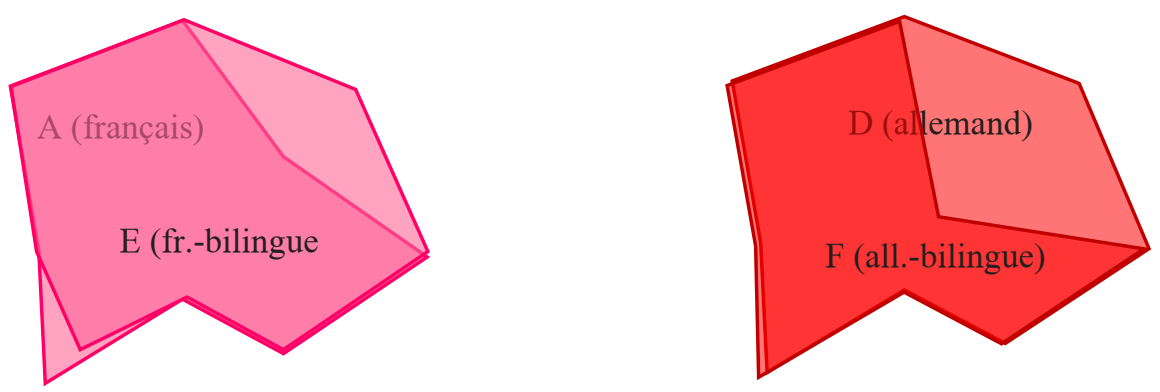
Fig. 18: Identification de congruence comportementale intra-individuelle: les cas des professionnels $\mathrm{A} / \mathrm{E}$ et $\mathrm{D} / \mathrm{F}$

Pour conclure, nous pouvons revenir sur la prise de conscience formulée au début de notre article et considérer que l'émotion joue un rôle pivot dans "l'émergence du désaccord interculturel » [95]. Dans cette optique, il est impératif d'en apprendre plus sur la manière dont on gère les émotions dans des structures bi-plurilingues pour améliorer les processus co-régulatifs et ceci pour les enfants autant que pour les professionnels. Le fait de disposer à présent d'un modèle qui permet de comparer le vécu des émotions et leur traitement in situ et cela même à un âge précoce, nous paraît prometteur et encourageant, surtout si on ne veut pas succomber à la reconstruction des stéréotypes culturels. Des adaptations au contexte adulte pourront et devront, bien sûr, suivre.

\section{Bibliographie}

1. H.M. Weiss, R. Cropanzano, Affective events theory : A theoretical discussion of the structure, cause and consequences of affective experiences at work, Res. Organ. Behav., 18, 1-74 (1998)

2. A.Th. WAN, How Can Learners Learn from Experience? A Case Study in Blended Learning at Higher Education, IJIET, 5, 8, 615-619 (2015).

3. G. Gebauer, M. Holodynski, S. Koelsch, Ch. Von Scheve, Von der Emotion zur Sprache : Wie wir lernen, über unsere Gefühle zu sprechen, Velbrück: Wissenschaft (2017)

4. N. Lim, Cultural differences in emotion: differences in emotional arousal level between the East and the West, IMR, 5, 2, 105-109 (2016).

5. J.-M. Dewaele, L. M. Pavelescu, The relationship between incommensurable emotions and willingness to communicate in English as a foreign language : a multiple case study, Innovat. Lang. Learn. Teach., 1-15 (2019). DOI:

6. A. Peña-Sarrionandia, M. Mikolajczak, J.J. Gross, Integrating emotion regulation and emotional intelligence traditions: a meta-analysis, Front. Psychol., 6 (2015)

7. Ch. Puren, Interculturalité et interdidacticité dans la relation enseignement-apprentissage en didactique des langues-cultures, Ela, 140, 491-512 (2015).

8. P. Gobin, A.-M. Camblats, W. Faurous, S. Mathey, Une base de l'émotionalité (valence, arousal, catégories) de 1286 mots français selon l'âge (EMA), Eur. Rev. Appl. Psychol., 67, 1 : 25-42 (2017).

9. I. Humair, A. Palama, E. Gentaz, Cross-modal recognition of emotion expressions from voice to face in 6-month-olds, Conference: 2nd Jean Piaget Conferences: Infant development from Piaget to today at Geneva, Switzerland (Genève, 2016)

10. A. Palama, L. Calpe, Ch. Fiorentini, J. Malsert, E. Gentaz, Does listening to an emotional voice (joy vs. anger) help their facial expression recognition in 4-month old? Research on audio-visual transfer Stimuli Participants, Unpublished (Poster sur ResearchGate, 2015).

11. A. Syssau, C. Monnier, L'influence de la valence émotionnelle positive des mots sur la mémoire des enfants, Psychol. Fr., 57, 4, 237-250 (2012).

12. A. Syssau, C. Monnier, Children's emotional norms for 600 French words, Behav. Res. Methods, 41, 1, 213-219 (2009).

13. W. Qu. Yow, E. M. Markman, Bilingualism and children's use of paralinguistic cues to interpret emotion in speech, BIL-LANG COGN, 14, 4, 562-569 (2011).

14. R. Sprengelmeyer, A. Calder, P. Ekman, A. Young, D. Perrett, Facial expressions of emotion: Stimuli and tests (FEEST). Psychology manual v1.0 Thames Valley Test Company. (Bury St Edmunds, England, 2002).

15. Denham (1986: 197) S.A. Denham, Social Cognition, Prosocial Behavior, and Emotion in Preschoolers: Contextual Validation, Child Dev., 57, 1, 194-201 (1986).

16. S.A. Denham, H. H. Bassett, Ch. Brown, E. Way, J. Steed, "I Know How You Feel”: Preschoolers' emotion knowledge contributes to early school success, Early Child. Res. Q., 13, 3, 252-262 (2015).

17. P. Ferré, M. Guasch, C. Moldovan, R. Sánchez-Casas, Affective norms for 380 Spanish words belonging to three different semantic categories, Behav. Res. Methods, 44, 2, 395-403 (2012). 
18. E.A. Walle, P.J. Reschke, L.A. Camras, J. Campos, Infant differential behavioral responding to discrete emotions, Emotion, 17, 7, 1078-1091 (2017).

19. G. Chronaki, M. Wigelsworth, M. D. Pell, S.A. Kotz, The development of cross-cultural recognition of vocal emotion during childhood and adolescence, Sci. Rep., 8,1 (2018).

20. V.L. Castro, L.A. Camras, A.G. Halberstadt, M. Shuster, Children's prototypic facial expressions during emotion-eliciting conversations with their mothers, Emotion, 18 ,2, 260-276 (2018).

21. D. Galati, B. Sini, Les structures sémantiques du lexique français des émotions. Dans Ch. Plantin, M. Doury, V. Traverso, Les émotions dans les interactions, 75-87 (Lyon, Presses Universitaires, 2000)

22. I. Novakova, A. Tutin (dirs.), Le lexique des émotions. (Grenoble, ELLUG, Université Stendhal, 2009)

23. P. Blumenthal Peter, Les noms d'émotion: Trois systèmes d'ordre. Dans I. Novakova, A. Tutin, Le lexique des émotions, 41-64, (Grenoble : ELLUG, Université Stendhal)

24. C. Monnier, A. Syssau, Affective norms for french words (FAN), Behav. Res. Methods, 46 , 4, 1128-1137 (2014).

25. S. Diwersy, O. Kraif, Observations statistiques de cooccurrents lexico-syntaxiques pour la catégorisation sémantique d'un champ lexical. Dans F. Baider, G. Cislaru, Cartographie des emotions: propositions linguistiques et sociolinguistiques, 55-69 (Paris, Presses Sorbonne Nouvelle, 2013)

26. A. Grutschus, A. Kern, A. Tutin, La polarité du lexique de l'affect: perspective combinatoire et contrastive. Dans F. Baider, G. Cislaru, Cartographie des emotions: propositions linguistiques et sociolinguistiques. 85-96, (Paris, Presses Sorbonne Nouvelle, 2013).

27. A. Polguère, Les petits soucis ne poussent plus dans le champ lexical des sentiments. Dans F. Baider, G. Cislaru, Cartographie des emotions: propositions linguistiques et sociolinguistiques, 21-41 (Paris, Presses Sorbonne Nouvelle, 2013).

28. G. Hassler, Synonymie et incompatibilité des noms d'émotions, Lidil, 32, 1-11 (2005).

29. G.L. Clore, A. Ortony, The Semantics of the Affective Lexicon. Dans V. Hamilton, G.H. Bower, N.H. Frijda, Cognitive Perspectives on Emotion and Motivation, 367-397 (Dordrecht, Springer Netherlands, 1988)

30. M. Quignard, B. Ursi, N. Rossi-Gensane, V. André, H. Baldauf-Quilliatre, C. Etienne, Ch. Plantin, V. Traverso, Une méthode instrumentée pour l'analyse multidimensionnelle des tonalités émotionnelles dans l'interaction, SHS Web Conf., 27, 1-19.

31. Ch. Plantin, Les bonnes raisons des émotions: Principes et méthode pour l'étude du discours émotionnél, (New. Bern, Peter Lang, 2016)

32. Ch. Plantin, M. Doury, V. Traverso, Les émotions dans les interactions, (Lyon, Presses Universitaires, 2000)

33. C. Polo, Ch. Plantin, K. Lund, G. Niccolai, Quand construire une position émotionnelle, c'est choisir une conclusion argumentative : le cas d'un café-débat sur l'eau potable au Mexique1, Semen, 35, 41-64 (2013).

34. J. Cosnier Jacques, Expression et régulation des émotions dans les interactions de la vie quotidienne, Colloque international sur les émotions, Paris : Laboratoire européen de psychologie sociale des Sciences de l'Homme, 1-23 (1987).

35. G.L. Clore, A. Ortony, Cognition in Emotion: Always, Sometimes, or Never?. Dans R.D. Lane, L. Nadel, Cognitive Neuroscience of Emotion, 24-61 (Oxford, Oxford University Press, 2002)

36. E. Nagy, P. Molnar, Homo imitans or homo provocans? Human imprinting model of neonatal imitation, Infant Behav. Dev., 27, 1, 54-63 (2004).

37. J.-M. Dewaele, Multi-competence and Emotion. Dans L. Wei, V. Cook, The Cambridge Handbook of Linguistic Multi-Competence, 461-477 (Cambridge, Cambridge University Press, 2016)

38. B.B. Schieffelin, Langue et lieu dans l'univers de l'enfance, Anthropol Soc., 31, 1, 15-37 (2007).

39. C. Trevarthen, Making Sense of Infants Making Sense, Intellectica, 34,1, 161-188 (2002).

40. A. Ortony, G.L. Clore, A. Collins, The cognitive structure of emotions (Cambridge, Cambridge Univ. Press, 1999)

41. M. Bradley, P.J. Lang, Measuring Emotion: Behavior, Feeling, and Physiology. [dans R.D. Lane, L. Nadel, Cognitive Neuroscience of Emotion, 242-276 (Oxford University Press, 2002)

42. J.J. Gross, R.A. Thompson, Emotion Regulation. Conceptual Foundations. Dans J.J. Gross, Handbook of Emotion Regulation, 3-24 (New York, Guilford Press, 2007) 
43. M. Lajante, O. Droulers, Importation de la théorie de l'évaluation cognitive et dynamique des processus émotionnels : illustration par la mesure de l'activité du système nerveux autonome, Management \& Avenir, 62,4, 171-187 (2013).

44. A. Ortony, G.L. Clore, Emotions, moods, and conscious awareness; comment on Johnson-Laird and Oatley's "The language of emotions: An analysis of a semantic field", Cogn. Emot., 125-137 (1989).

45. K.N. Ochsner, J.J. Gross, The Neural Architecture of Emotion Regulation. Dans J.J. Gross, Handbook of Emotion Regulation, 87-109 (New York, Guilford Press, 2007)

46. A.R. Damasio, Descartes' error: emotion, reason and the human brain (London, Vintage, 2006)

47. J.S. Bruner, Le développement de l'enfant : savoir faire, savoir dire (Paris, PUF, 1983)

48. J.S. Bruner, Comment les enfants apprennent à parler (Paris, Retz, 1987)

49. I.B. Mauss, R.W. Levenson, L. McCarter, F.H. Wilhelm, J.J. Gross, The Tie That Binds? Coherence Among Emotion Experience, Behavior, and Physiology, Emotion, 5, 2, 175-190 (2005).

50. D. Grandjean, D. Sander, K.R. Scherer, Conscious emotional experience emerges as a function of multilevel, appraisal-driven response synchronization, Conscious. Cogn., 17, 2, 484-495 (2008).

51. J.-F. Moulin, Le discours silencieux du corps enseignant: La communication non verbale du maître dans les pratiques de classe, Carrefours de l'éducation, 17, 1, 142-159 (2004).

52. L. Devillers, M. Tahon, M.A. Sehili, A. Delaborde, Détection des états affectifs lors d'interactions parlées : robustesse des indices non verbaux, TAL, 55, 2,123-149 (2014).

53. J. Abitbol, L'odyssée de la voix (Paris, Robert Laffont, 2005)

54. R. Prat, Prendre une voix dans ses bras, Enfances \& Psy, 58, 1, 29-39 (2013)

55. E.T. Hall, La dimension cachée (Paris, Points Essai, 1971)

56. J. Grapperon, A.-C. Pignol, J.Vion-Dury, La mesure de la réaction électrodermale, Encephale, 38, 2, 149-155 (2012).

57. L. Chaiguerova, Note sur les traductions. [dans F. Yvon et Y. Zinchenko, Vygotsky, une théorie du développement et de l'éducation : recueil de textes et commentaires, 59-62 (Moscou, Faculté de psychologie de l'Université d'État de Mouscou Lomonossov, 2011)

58. F. Yvon et Y. Zinchenko, Vygotsky, une théorie du développement et de l'éducation : recueil de textes et commentaires (Moscou, Faculté de psychologie de l'Université d'État de Mouscou Lomonossov, 2011)

59. D. Sorter, B. Beebe, J. Rustin, S. Knoblauch, A Comparison of Meltzoff, Trevarthen, and Stern, Psychoanal. Dialogues, 13, 6, 777-804 (2003).

60. A.R. Damasio, C. Larsonneur, Le sentiment même de soi : corps, émotions, conscience (Paris, O. Jacob, 1999)

61. Y. Gervais, Comprendre la base de la communication non verbale. Silence! Moteur! Action! INITIATION AU CINÉMA pour le primaire (2017).

62. P. García-Prieto, V. Tran, T. Wranik, Les théories de l'évaluation cognitive et de la différenciation des émotions: une clé pour comprendre le vécu émotionnel au travail. Dans N. Delobbe, O. Herrbach, D. Lacaze, K. Mignonac, Comportement organisationnel, 195-222 (Bruxelles, De Boeck, 2005)

63. N.H. Frijda, Emotion Experience and its Varieties, Emot. Rev., 1,3, 264-271 (2009).

64. A. Celeghin, M. Diano, A. Bagnis, M. Viola, M. Tamietto, Basic Emotions in Human Neuroscience: Neuroimaging and Beyond, Front. Psychol., 8, Article 1432, 1-13 (2017).

65. J.R.J. Fontaine, K.R. Scherer, E.B. Roesch, Ph.C. Ellsworth, The World of Emotions is not TwoDimensional, Psychol. Sci., 18,12, 1050-1057 (2007).

66. R.A. Thompson, S. Meyer, Socialization of Emotion Regulation in the Family. Dans J.J. Gross, Handbook of Emotion Regulation, 249-268 (New York, Guilford Press, 2007)

67. L. Nummenmaa, H. Saarimäki, Emotions as discrete patterns of systemic activity, Neurosci. Lett., 693, 3-8 (2019).

68. Kerbrat-Orecchioni, Catherine, Les interactions verbales, Tome 1 (Paris, Éditions Armand Colin, 1990)

69. Kerbrat-Orecchioni, Catherine, La notion d'interaction en linguistique : origine, apports, bilan, Lang. Francaise, 117, 1, 51-67 (1998).

70. F. Berthelon, Modélisation et détection des émotions à partir de données expressives et contextuelles (Université Nice Sophia Antipolis, 2013). 
71. R. Sprengelmeyer, A.W. Young, A.J. Calder, A. Karnat, H. Lange, V. Homberg, D.I. Perrett, D. Rowland, Loss of disgust. Perception of faces and emotions in Huntington's disease, Brain, 119, 1647-1665 (1996).

72. P. Ekman, W.V. Friesen, Pictures of facial affect, Palo Alto (CA): Consulting Psychologists Press (1976)

73. C. Daudelin-Peltier, L'impact d'un stress psychosocial sur la reconnaissance des expressions faciales d'émotion (Gatineau, Université du Québec en Outaouais, 2016)

74. K.R. Scherer (2005). What are emotions? And how can they be measured?, Soc. Sci. Inf., 44, 4, 695-729.

75. R. Micheli, I. Hekmat, A. Rabatel, Les émotions : des modes de sémiotisation aux fonctions argumentatives, Semen, 35, 7-16 (2013).

76. K. Kwapisz-Osadnik, Katarzyna, Entre universalisme cognitif et relativisme culturel : expression des émotions en polonais et en français dans un cadre de la linguistique cognitive/ Between cognitive universalism and cultural relativism: expressing emotions in Polish and French 2017) Dans F. Baider, G. Cislaru, Cartographie des émotions: propositions linguistiques et sociolinguistiques , 151-163 (Paris, Presses Sorbonne Nouvelle, 2017)

77. A. Pavlenko, Emotions and multilingualism (Cambridge/New York, Cambridge University Press, 2005)

78. A.-L. Gilet, D. Grühn, J. Studer, G. Labouvie-Vief, Valence, arousal, and imagery ratings for 835 French attributes by young, middle-aged, and older adults: The French Emotional Evaluation List (FEEL), Eur. Rev. Appl. Psychol., 62, 3, 173-181 (2012).

79. M. Yik, J.A. Russell, J.H. Steiger, A 12-point circumplex structure of core affect, Emotion, 11, 4, 705-731 (2011).

80. V. Sacharin, K. Schlegel, K.R. Scherer, Geneva Emotion Wheel Rating Study (Genève, archive ouverte UNIGE, 2012).

81. Ch. Plantin, N. Tersis, Les émotions dans la langue et les chants inuit. Dans N. Tersis, P. Boyeldieu, Le langage de l'émotion : Variations linguistiques et culturelles, 93-122 (Leuven, Peeters, 2017)

82. L.-G. Lundh, Placebo, belief, and health. A cognitive-emotion model, Scand. J. Psychol., 28, 2, 128-143 (1987).

83. R. Micheli, Esquisse d'une typologie des différents modes de sémiotisation verbale de l'émotion, Semen, 35, 17-39 (2013).

84. L. Lacaze, L'interactionnisme symbolique de Blumer revisité, Sociétés, 121, 3, 41-52 (2013).

85. H. Blumer, Herbert, Symbolic Interactionism: Perspective and Method (Berkeley/Los Angeles/ London, University of California Press, 1969)

86. A. Todorov, L.T. Harris, S.T. Fiske, Toward socially inspired social neuroscience, Brain Res., 1079, 1, 76-85 (2006).

87. L. Feldman Barrett, How Emotions are Made: The Secret Life of the Brain (New York, Houghton Mifflin Harcourt, 2017)

88. A.R. Hochschild, Emotion Work, Feeling Rules, and Social Structure, Am. J. Sociol., 85, 3, 551-575 (1979).

89. M. Richelle, Analyse formelle ou analyse fonctionnelle du comportement verbal. Notes sur le débat entre Chomsky et Skinner, B. psychol., 26, 252-259 (1972).

90. B. Elsner, Theorien zu Handlungsverständnis und Imitation. Dans L. Ahnert, Theorien in der Entwicklungspsychologie, 310-329 (Berlin Heidelberg, Springer VS Lehrbuch, 2014)

91. G. Rizzolatti, L. Cattaneo, M. Fabbri-Destro, S Rozzi, Cortical Mechanisms Underlying the Organization of Goal-Directed Actions and Mirror Neuron-Based Action Understanding, Physiol. Review, 94, 2, 655-706 (2014).

92. A. Ittel, D. Raufelder, H. Scheithauer, Soziale Lerntheorien. Dans L. Ahnert, Theorien in der Entwicklungspsychologie, 330-353 (Berlin Heidelberg, Springer VS Lehrbuch, 2014)

93. E. Hatfield, J.T. Caccioppo, R.L. Rapson, Emotional contagion (Cambridge, Cambridge University Press, 1994)

94. E. Morin, La méthode (Paris, Édition du Seuil, 2014)

95. A. Wagener, L'émotion dans l'émergence du désaccord interculturel. Dans B. Pothier, Langue, langage et interactions culturelles, 133-153 (Paris, L'Harmattan, 2009) 